\title{
Experiments on transient melting of tungsten by ELMs in ASDEX Upgrade
}

\author{
K. Krieger ${ }^{1}$, M. Balden ${ }^{1}$, J.W. Coenen ${ }^{2}$, F. Laggner ${ }^{4}$, G.F. Matthews ${ }^{3}$, D. Nille ${ }^{1}$, V. Rohde ${ }^{1}$, B. Sieglin ${ }^{1}$, \\ L. Giannone ${ }^{1}$, B. Göths ${ }^{2}$, A. Herrmann ${ }^{1}$, P. de Marne ${ }^{1}$, R.A. Pitts ${ }^{5}$, S. Potzel ${ }^{1}$, P. Vondracek ${ }^{6}$, \\ ASDEX-Upgrade Team ${ }^{1}$, EUROfusion MST1 Team ${ }^{*}$ \\ ${ }^{I}$ Max-Planck-Institut für Plasmaphysik, 85748 Garching b. München, Germany \\ ${ }^{2}$ Forschungszentrum Jülich GmbH, Institut für Energie- und Klimaforschung - Plasmaphysik, \\ 52425 Jülich, Germany \\ ${ }^{3}$ Culham Centre for Fusion Energy, Abingdon, Oxon, OX14 3DB, United Kingdom \\ ${ }^{4}$ TUWien,Fusion@ÖAW,1040Wien,Austria \\ ${ }^{5}$ ITER Organization, Route de Vinon, CS 90 046, 13067 Saint Paul Lez Durance, France \\ ${ }^{6}$ Institute of Plasma Physics AS CR, Za Slovankou 1782/3, 18200 Praha 8, Czech Republic \\ "See the author list of "H. Meyer et al 2017 Nucl. Fusion 57 102014"
}

\begin{abstract}
Repetitive melting of tungsten by power transients originating from edge localized modes (ELMs) has been studied in ASDEX Upgrade. Tungsten samples were exposed to H-mode discharges at the outer divertor target plate using the Divertor Manipulator II (DIM-II) system [1]. Designed as near replicas of the geometries used also in separate experiments on the JET tokamak [2-4], the samples featured a misaligned leading edge and a sloped ridge respectively. Both structures protrude above the default target plate surface thus receiving an increased fraction of the parallel power flux. Transient melting by ELMs was induced by moving the outer strike point to the sample location. The temporal evolution of the measured current flow from the samples to vessel potential confirmed transient melting. Current magnitude and dependency from surface temperature provided strong evidence for thermionic electron emission as main origin of the replacement current driving the melt motion. The different melt patterns observed after exposures at the two sample geometries support the thermionic electron emission model used in the MEMOS melt motion code, which assumes a strong decrease of the thermionic net current at shallow magnetic field to surface angles [5]. Post exposure ex-situ analysis of the retrieved samples show recrystallization of tungsten at the exposed surface areas to a depth of up to several $\mathrm{mm}$. The melt layer transport to less exposed surface areas leads to ratcheting pile up of re-solidified debris with zonal growth extending from the already enlarged grains at the surface.
\end{abstract}

Keywords: plasma facing components, tungsten, melting.

\section{Introduction}

The use of tungsten as material for the ITER divertor target plates introduces the risk of melt damage by excessive heat loads. These may occur by either in steady state due, for example to divertor reattachment events or in transient situations as a result of insufficiently controlled/mitigated disruptions (during the thermal and current quench and/or as a result of runaway electrons) and edge localized modes (ELMs) [5]. The latter mechanism can occur repeatedly over a succession of subsequent ELMs with corresponding progression of surface damage. During each melt event, molten tungsten may be redistributed by electromagnetic, hydrodynamic and gravitational forces acting on the fluid phase. The resulting increasing surface corrugation is likely to decrease the power handling capability of the affected target plate [6]. Moreover, there are risks to plasma operation as a consequence of released melt droplets during the melt event as well as by potential spallation of larger masses of re-solidified material piled up in regions of low power flux exposure by melt flow along the divertor surface. The potentially severe consequences of melt events for ITER operation have motivated detailed investigations using both predictive computer simulations and experiments on present day tokamaks [5].

The main tool for modelling of melt motion at metallic plasma facing components is the 3D MEMOS (Melt Motion at Surfaces) code described in [7, 8]. To validate the MEMOS code model for tokamak conditions, tungsten melting and resulting melt motion were first studied in TEXTOR [9, 10]. In these experiments a tungsten test limiter was exposed to the hot boundary plasma with a power flux at the surface sufficient to induce sustained melting over a time of several seconds. The melt motion observed on the heated surface could be quantitatively reproduced by corresponding MEMOS simulations [11, 12]. From the MEMOS results the main driver of melt motion was identified as the $\mathrm{j} \times \mathrm{B}$ force originating from the interaction of the thermionic emission current through the heated surface with the plasma confining magnetic 
field. A similar experiment was carried out in ASDEX Upgrade [13] where a sample with misaligned castellation cells was exposed in the outer divertor using the divertor manipulator I system [14]. Melting of the misaligned edge was induced by moving the outer strike point (OSP) to the position of the sample. Similar to the TEXTOR experiments, the melt motion could be interpreted as result of $\mathrm{j} \times \mathrm{B}$ and gravitational force with the electromotive force being the dominant mechanism. Liquid tungsten was also observed to flow across the castellation cell gaps and re-solidify at the cooler surface of the surrounding tile. By spallation of a fraction of that weakly attached debris perpendicular to the divertor plate surface in the following discharge, a tungsten fragment could reach the confined plasma leading to a disruption [13], which confirmed the potential risk of melt events for plasma operation. In contrast, the ejection of droplets also observed in the experiment did not significantly affect plasma performance because the droplets would generally follow trajectories toroidally within the divertor over meter distances without entering the confined plasma region [14-16].

All these results referred to the case of tungsten bulk melting over intervals of several seconds and therefore cannot be directly applied to the case of repeated transient melting by ELMs, in which the heat pulse duration occurs on the $\approx 1 \mathrm{~ms}$ timescale. In contrast to sustained melting over a timescale of seconds where melting can extend to $\mathrm{mm}$ depths, transient melting drives typical melt layer thicknesses in the $10 \mu \mathrm{m}$ range [8]. Due to the rapid re-solidification, lateral melt transport away from the actual melt zone is expected to occur mainly in incremental steps during repeated transients.

To extend the validation of the MEMOS model to the case of repetitive transient melting of tungsten by ELMs, an experiment was first carried out in the JET tokamak by modifying one of the bulk tungsten outer divertor modules [2-4]. The modified tile included a special tungsten lamella designed to provide a leading edge facing the full parallel power flux. This was achieved by decreasing the height of eight preceding upstream lamellae by $\approx 1 \mathrm{~mm}$. Transient melting along the leading edge by ELMs was induced in six identical and sequential H-mode discharges. The melt events were subsequently modelled with MEMOS-3D. The key input parameter for the simulations is the parallel power flux along the lamella as function of time, which was derived from infrared (IR) thermography measurements. The main observables to be matched against the simulations were the measured lamella surface temperature and the shapes of the melt zone and re-solidified melt debris. Because the IR camera viewed the divertor module from top, it could not directly detect the temperature at the melting surface. Instead the local temperature distribution had to be derived by interpretative modelling [17]. To match the IR data, an ad hoc reduction factor was initially assumed for the parallel heat flux to the leading edge [3]. It has since been possible to understand the origin of this apparent deficit through more sophisticated thermal transport models and the use of corrections taking into account the local exposure geometry $[18,19]$.

The validity of the geometric projection for the calculation of the parallel power flux at JET, called into question by the first leading edge experiment, was tested experimentally in a second transient melt study using an alternative lamella design with a protruding sloped surface facing the toroidal field allowing direct IR observation of the slope surface [20]. The conclusion of this work, and of the detailed new thermal analysis [19], is that the optical approximation (i.e. in which finite ion Larmor orbits are not accounted for) is valid at JET to a good approximation. This has also been the conclusion of a wider multi-machine study conducted under the auspices of the International Tokamak Physics Activity (ITPA), of which the JET experiments and those reported here are a part [5].

Using the IR measured parallel power flux profile as input, the MEMOS code has been extremely successful in reproducing quantitatively the observed erosion and melt motion seen in the JET experiment $[2,5]$. In common with the TEXTOR experiments $[11,12]$ the MEMOS simulations identified the $\mathrm{j} \times \mathrm{B}$ force originating from thermionic electron emission as the main driving force of the melt motion in the JET experiment $[2,3,5$, 20]. However, direct validation of the thermionic emission model used in MEMOS was not possible because in JET the actual current could not be directly measured. This left a number of uncertainties resulting from the range of published data for the tungsten work function and from assumptions on the possible attenuation of the emission current by space charge effects and locally returning electrons [5]. To overcome this limitation, a corresponding experiment, described in this paper, was planned in ASDEX Upgrade with samples designed to allow direct measurement of the replacement current driven by thermionic emission.

\section{Experimental set up}

\subsection{Sample arrangement and instrumentation}

Experiments which involve deliberate melting of plasma facing components are hampered by the requirement that the damaged components must not interfere with subsequent plasma operation. In JET, the only way to avoid this was to install the special lamella samples in the innermost ring of the segmented bulk tungsten divertor plate, which is almost never solicited during 
normal plasma operations. In the ASDEX Upgrade experiment the samples were instead exposed using its Divertor Manipulator II system (DIM-II) [1] and retrieved immediately after the experiment for ex-situ materials analysis. As shown in Fig. 1, DIM-II allows two adjacent standard sized outer divertor target tiles to be exposed. Access to the tiles and samples/ instrumentation they carry is possible by retracting the entire tile assembly mounted on the manipulator arm behind the vessel port valve. With the valve closed the system can be vented without breaking the torus vacuum. Due to the time required to pump down the external volume to torus vacuum, sample exchange is only possible in between experiment days.

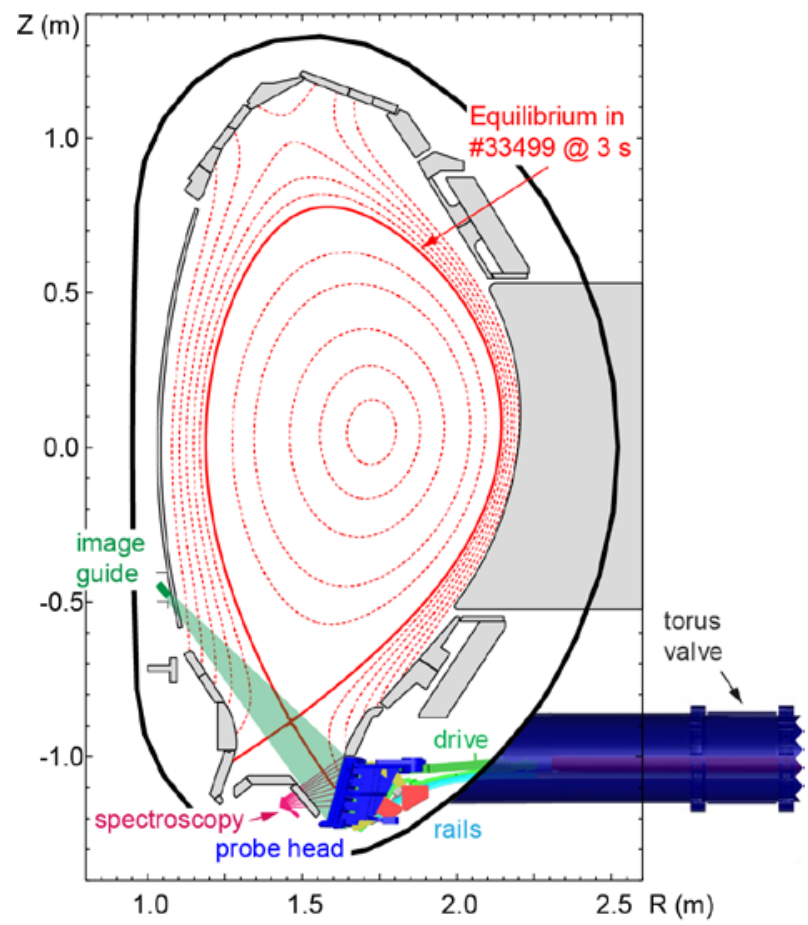

Fig. 1 Poloidal cross-section of the ASDEX Upgrade vessel with divertor manipulator II structure [21] and flux surfaces of reference H-mode discharge \#33499 in the flat top phase at $t=3 \mathrm{~s}$ with the outer strike point at the position preceding sample exposure.

By default the manipulator is equipped with two standard bulk tungsten target tiles. For the melt experiment these were replaced by instrumented tiles (see Fig. 2) designed with electrically insulated holding fixtures and sample contacts connected to electrical feedthroughs for air side current measurement. The current flowing to vessel ground was determined from the voltage drop across shunt resistors with a sampling rate of $200 \mathrm{kHz}$. In addition, the tiles were equipped with a mantle thermocouple for each sample, pressed to the sample rear side by a coil spring. The thermo-voltage was recorded with a sampling rate of $1 \mathrm{kHz}$ over a time interval of $200 \mathrm{~s}$ during and after discharges for calorimetric quantification of the total energy input to the sample. The outer jacket of the thermocouples was also electrically insulated so as not to interfere with the sample current measurement. For direct comparison to the JET melt experiments the elevated features of the samples were designed with cross-sectional dimension identical to those of the JET special lamellas (see Fig. 3).

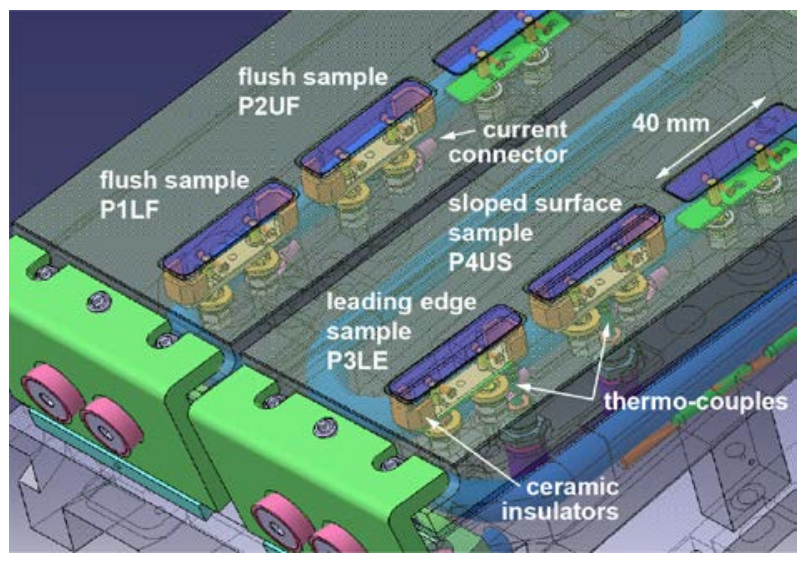

Fig. 2 CAD view of the two instrumented tiles mounted on the DIM-II probe head [21] with installed samples, ceramic insulation, electrical contact points and mantle thermocouples.

As shown in Fig. 2, the samples to be exposed in the experiment were installed in the two lower slots of the right hand tile of the manipulator head (seen from plasma side). For reference, flat samples were installed flush to the tile surface in the left hand tile. The flat samples installed in the upper slots were not used in the experiment.
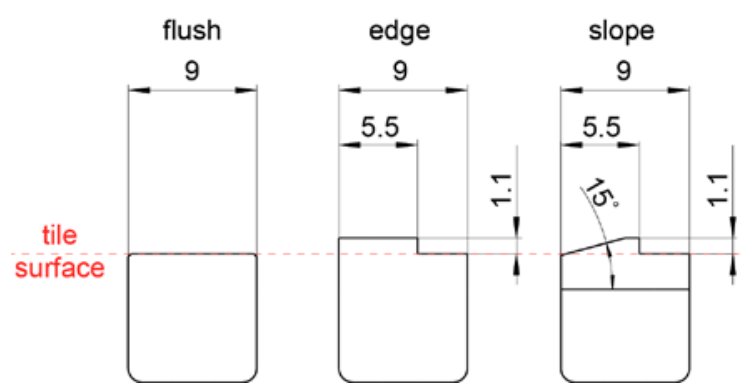

Fig. 3 Toroidal cross-section of the bulk tungsten sample inserts (dimensions in $\mathrm{mm}$ ) [21].

\subsection{Discharge scenario}

Given typical ELM plasma energy losses, $\Delta W_{\text {ELM }}$ in the range of a few tens of $\mathrm{kJ}$ on ASDEX Upgrade, to achieve repetitive ELM-induced transient tungsten melting requires sufficient sample exposure time to pre-heat the samples to a suitable base temperature. In between ELMs the surface temperature must be sufficiently close to the melting temperature of tungsten at $3695 \mathrm{~K}$ that melting will occur by the additional temperature excursion due to the ELM power load. At the same time, the average power input to the samples in the exposure time interval must be low enough to avoid the onset of sustained bulk melting. Optimal conditions will therefore 
be met by an H-mode discharge tailored for maximum Type-I ELM parallel power flux $\left(\approx 1 \mathrm{GW} / \mathrm{m}^{2}\right)$ and low ELM frequency, $f_{\mathrm{ELM}}$. However, for the melt experiment an additional boundary condition is stability of the plasma configuration against the sweeps of the OSP which are required to control the plasma exposure of the melt samples.

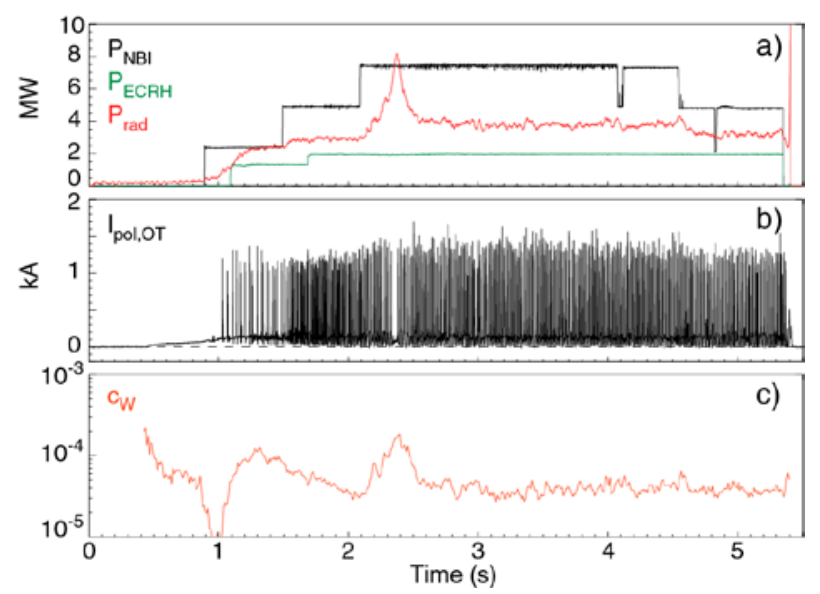

Fig. 4 Reference H-mode discharge \#33499 without OSP sweep. The time traces show (a) auxiliary heating power, $P_{N B I}$ and $P_{E C R H}$, and total radiated power, $P_{\text {rad }}$, computed from bolometer arrays; (b) the poloidal (mostly thermoelectric) current to the outer target plate as ELM signature; (c) the central tungsten concentration, $c_{W}$.

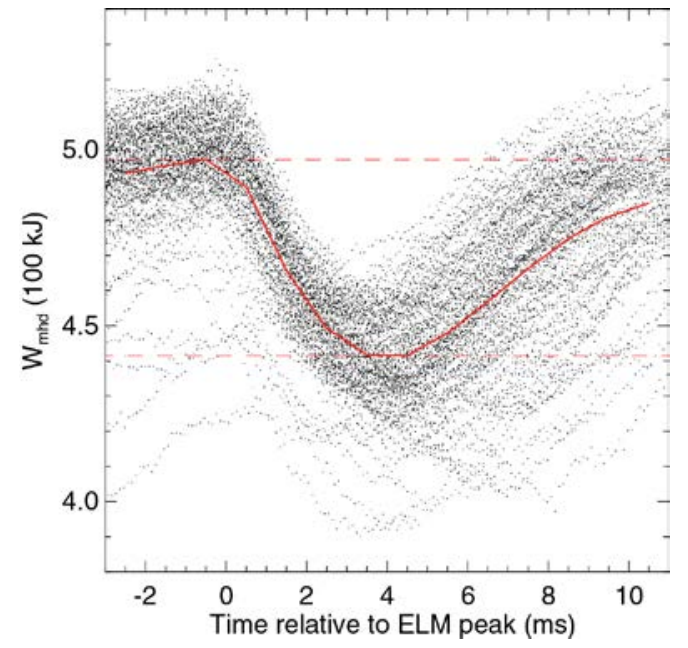

Fig. 5 Coherent average of plasma stored energy in discharge 33499 over all ELMs in the time interval from 2.6-4.4 s where NBI heating power was constant at 7.5 MW. The average ELM energy was determined as the difference between the maximum plasma stored energy just before the ELM onset and the minimum value reached during the ELM crash (dashed red lines).

To satisfy these criteria, the melt experiment was performed using a H-mode discharge scenario developed for pedestal and ELM studies [22, 23] with a plasma current of $I_{p}=0.8 \mathrm{MA}$, a toroidal field of $B_{t}=-2.5 \mathrm{~T}$, $\left(q_{95}=4.6\right)$ and a heating power of $7.5 \mathrm{MW}$ Neutral Beam Injection (NBI) and $2 \mathrm{MW}$ Electron Cyclotron
Resonance Heating (ECRH) (Fig. 4). The central ECRH was applied to maintain a stationary tungsten concentration during the flat top phase at a level of $c_{W} \approx 4 \times 10^{-5}$ so that a potential transient tungsten source by droplet ejection during a melt event would be clearly visible.

At the chosen deuterium gas feed, $\mathrm{I}_{\mathrm{p}}$ and heating power, $f_{\text {ELM }} \approx 72 \mathrm{~Hz}$, with a corresponding average inter-ELM period of $14 \mathrm{~ms}$ and $\Delta W_{\mathrm{ELM}} \approx 55 \mathrm{~kJ}$, the latter determined by coherent averaging of the plasma stored energy over the ELMs during the plasma flat-top phase (see Fig. 5). This corresponds to $\approx 12 \%$ of the total stored plasma energy.

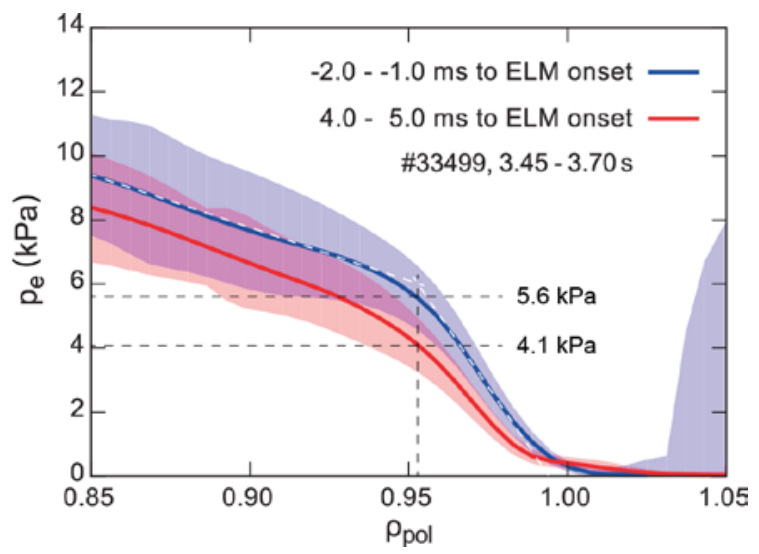

Fig. 6 Profile of the plasma electron pressure in the reference $\mathrm{H}$-mode transport barrier region, $p_{e}$, before and after the onset of an ELM as function of normalized radius, $\rho_{\mathrm{pol}}$.

With a pedestal top pressure of $p_{\text {ped }} \approx 5.6 \mathrm{kPa}$ (see Fig. 6) the scaling law established in $[24,25]$ yields an expected lower limit of the parallel ELM energy density of $\varepsilon_{\|}\left[\mathrm{MJ} / \mathrm{m}^{2}\right]=6 \pi p_{\text {ped }}[\mathrm{MPa}] \times R_{\text {geo }} \times q_{95} \approx 0.8 \mathrm{MJ} / \mathrm{m}^{2}$ reaching the outer divertor target plate (ASDEX Upgrade major radius, $R_{\text {geo }}=1.62 \mathrm{~m}$ ). This is $60 \%$ of the corresponding value in the JET experiment $\left(\varepsilon_{\|}=1.33 \mathrm{MJ} / \mathrm{m}^{2} \quad\right.$ with $\quad p_{\text {ped }} \approx 5.6 \mathrm{kPa}, \quad R_{\text {geo }}=2.9 \mathrm{~m}$, $\left.q_{95}=2.9\right)$ and in both the ASDEX Upgrade and JET cases is well above the transient melt threshold energy density (0.6-0.7 $\mathrm{MJm}^{-2}$ ) observed for ELM-like $0.5 \mathrm{~ms}$ heat pulses in quasistationary plasma accelerator (QSPA) experiments [26-28]. Since the scaling refers to the parallel energy density, the energy density on the sloped sample surface, $\varepsilon_{\text {surf, }}$ must be projected onto the surface taking into account the incident magnetic field angle and the angle of the slope. For a typical value of $\theta=18^{\circ}$ in the ASDEX Upgrade case $\left(15^{\circ}\right.$ slope $+\approx 3^{\circ}$ field line angle) $\varepsilon_{\text {surf }}=0.24 \mathrm{MJ} / \mathrm{m}^{2}$, however, taking into account that $\varepsilon_{\|}$can exceed the lower limit given in the scaling by a factor three [24], transient melting by ELMs may also be expected for this sample shape. In contrast to JET, the parallel ELM energy density in ASDEX Upgrade is still below the evaporation threshold for tungsten $\left(1.1 \mathrm{MJm}^{-2}\right.$, [28]) so that no vapour cloud formation and resulting 
power flux shielding effects are to be expected for the ASDEX Upgrade ELM conditions.

Following a reference H-mode discharge with the OSP maintained fixed above the exposure zone, the samples were exposed in subsequent discharges for successively increasing durations. To minimize sample exposure before the desired exposure conditions were established, the OSP was moved rapidly above the sample locations following X-point formation while at the same time ramping up the auxiliary heating power. To expose the samples, the OSP was then rapidly moved towards the sample position, held there for a programmed time interval, and moved back to the position above the samples before heating power rampdown and discharge termination (see Fig. 7).

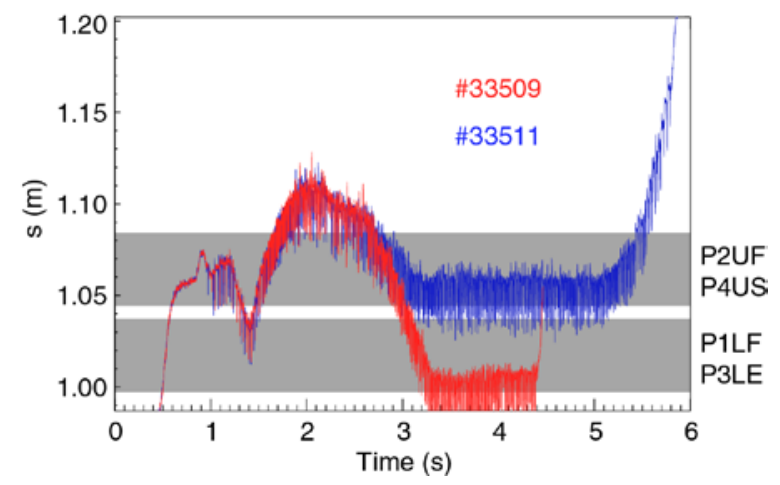

Fig. 7 Time traces of OSP position for exposure of lower samples (flush sample P1LF, leading edge sample P3LE) and upper samples (flush sample P2UF, sloped surface sample P4US) respectively. The divertor s-coordinate extends from lower to upper edge of the outer target tile. See Fig. 2 for sample naming convention.

To avoid accidental overheating leading to sustained bulk melting of the samples, the exposure interval was increased in incremental steps from discharge to discharge. The onset of melting was monitored by checking the movies recorded by the camera viewing the probe head surface after each exposure (see Fig. 1 and Section 5). Exposures of the leading edge sample P3LE began with a duration of $0.2 \mathrm{~s}$ with the OSP in its lowest position. The duration was then increased to $0.5 \mathrm{~s}, 0.6 \mathrm{~s}$ and finally to $0.9 \mathrm{~s}$. In the last exposure, ejection of individual droplets was seen in six of the recorded camera frames between $3.95 \mathrm{~s}$ and $4.4 \mathrm{~s}$, providing clear evidence of melting so that further exposures of that sample were stopped. For the sloped sample the first discharge was programmed for a $0.5 \mathrm{~s}$ exposure interval and subsequently increased to $1 \mathrm{~s}, 1.5 \mathrm{~s}$ and finally to the maximum possible time interval of $2 \mathrm{~s}$. Melt ejection was not observed during exposure of the sloped sample. Visual documentation of the sample surface in between discharges by retracting the probe head to a dedicated airlock chamber observation window was not possible at the time of the experiment because the required camera system was not yet commissioned. Therefore only the final state of the samples after conclusion of the experimental session is known.

\section{Post-exposure melt pattern}

\subsection{Qualitative comparison to JET melt study}

At the end of the melt experiment session the probe head was retracted from the machine to retrieve the melt samples for ex-situ analysis. As will be discussed further in Section 3.2, the sample morphology was documented by macro-photography and laser profilometry. In addition, both the surface and the bulk material were analyzed using focused ion beam cuts into the surface and metallographic analysis of cross-sections (Section 4).

Fig. 8 shows the overall melt patterns for the two sample geometries. Despite identical plasma conditions (except for the different OSP position adjusted to the respective sample location) there are significant differences between the two geometries. These differences can therefore only result from the different projected power flux at the plasma wetted surfaces and from the different magnetic field to surface intersection angles (Section 6). In case of the sloped sample (Fig. 8a) the surface has developed corrugated structures formed by re-solidified melt. However, melt motion was rather limited in contrast to that at the leading edge sample (Fig. 8b) where molten material moved along the exposed edge to form a re-solidified pattern extending to the lower edge of the sample with some material even spilling over the gap to the surface of the surrounding tile (see also Fig. 9d). The melt motion was towards and across the separatrix, which can be qualitatively understood by a driving $\mathrm{j} \times \mathrm{B}$ force due to the interaction of a thermionic emission current from the surface with the toroidal magnetic field [4]. This is identical to the observation in the previous JET transient ELM melting experiment [2-5]. Indeed, the melt pattern of the ASDEX Upgrade leading edge sample is strikingly similar to that of the JET leading edge lamella (Fig. 8c), providing strong evidence that the melt motion in both cases is driven by the same fundamental processes. 


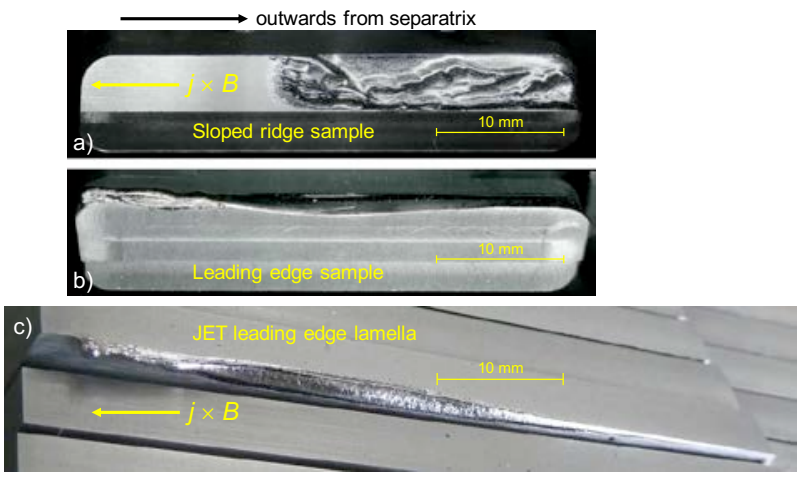

Fig. 8 Post exposure melt patterns of the ASDEX Upgrade sloped melt sample (a) and leading edge melt sample (b). For comparison the melt pattern after the JET leading edge lamella melt study [4] is shown with identical length scale (c).

As mentioned in Section 1, the melt motion observed in the JET experiment could be well reproduced by MEMOS simulations [3] with the thermionic emission current as the main melt motion driver. Further validation of the MEMOS model assumptions will be possible by the combined data set of melt motion morphology and direct current measurements of the ASDEX Upgrade melt study. Corresponding studies have already begun with first results published in [29].

\subsection{Quantitative analysis by $3 D$ profilometry}

Comparison of predicted melt motion with the simulations requires a quantitative mapping of the postexposure surface morphology. This was determined for both samples using a confocal 3D laser scanning microscope (Olympus OLS4000). As a consequence of the high reflectivity, particularly in the re-solidified surface areas, the samples were coated by spraying graphite powder immersed in a liquid onto the surface. Fig. $9 \mathrm{~b}$ and $\mathrm{c}$ show the surface scans across the plasma exposed parts of the leading edge sample from the side facing the parallel power flux (b) and from the top side receiving the projected power flux (c). By integration over the recessed surface fractions below the virgin surface level after melting, the melt surface area may be calculated as $\approx 12 \mathrm{~mm}^{2}$. The volume of melt moved away from the melt zone may be estimated at $6.8 \mathrm{~mm}^{3}$ from the top view (c) and $7.8 \mathrm{~mm}^{3}$ from the side view (b).

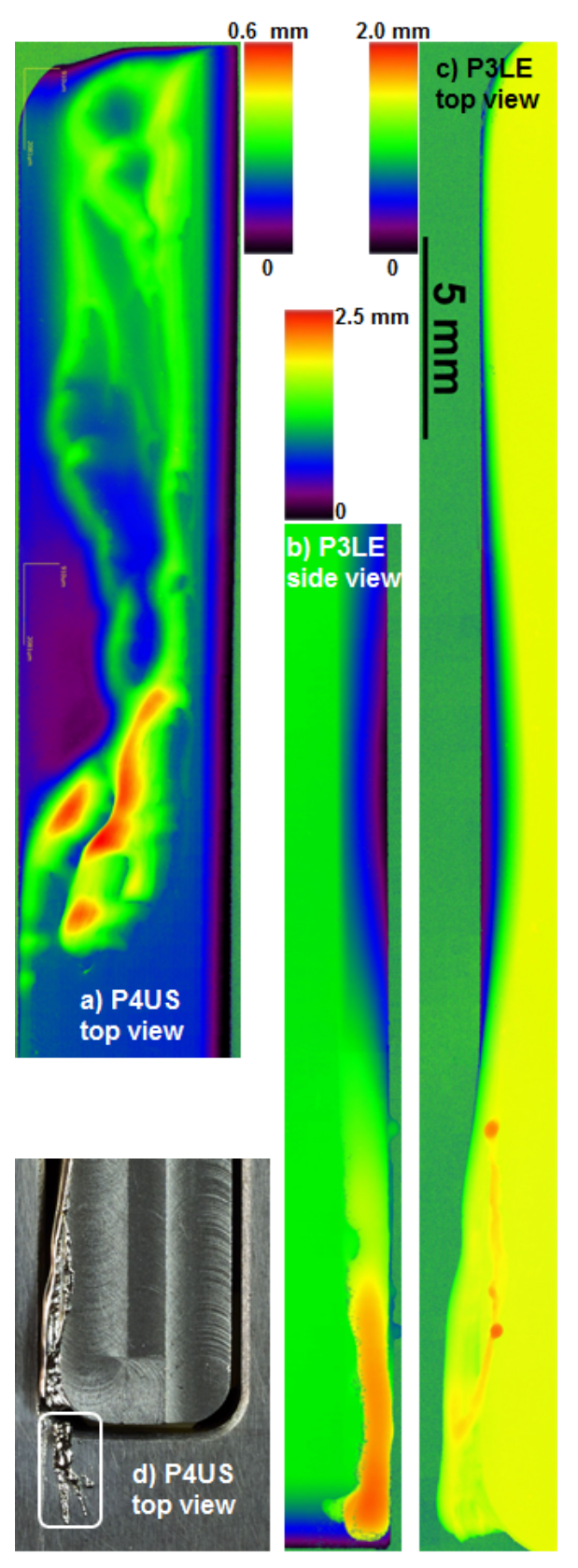

Fig. 9 Post-exposure surface morphology measured by a 3D laser scan in a confocal microscope. a) Molten area of sloped sample scanned from top (plasma side). b) Molten area of leading edge sample scanned from side at leading edge surface. c) Leading edge sample scanned from top (plasma side). d) Photo of resolidified melt and debris spilled over to surrounding frame tile.

Similarly the volume of melt residue piled up above the original surface level outside the melt zone was determined at $6.1 \mathrm{~mm}^{3}$. This yields a net loss of melt in the range of $0.7-1.7 \mathrm{~mm}^{3}$, equivalent to a mass of $13.5-$ $32.7 \mathrm{mg}$. The mass of the collected melt debris spilled over across the $0.5 \mathrm{~mm}$ gap to the surrounding frame tile surface (Fig. 9d), was determined by weighing to $15.9 \mathrm{mg}$. The bridge of tungsten melt between sample and frame tile was still in place at the end of the experiment. Due to the electrical short produced by the 
bridge, its formation time could be exactly determined from the probe current signal (see Section 6.1). The mass difference between the material loss from the melt zone and the collected melt residue sets an upper limit of $16.8 \mathrm{mg}$ for non-local melt losses, e.g. by melt ejection into the divertor plasma. As discussed in Section 2.2, some ejection events were indeed detected in the final leading edge sample exposure (discharge 33509) using the $\mathrm{CCD}$ camera directly viewing the probe surface. However, ejection of droplets with a total mass in the $\mathrm{mg}$ range can be excluded since droplets in that mass range have been observed to travel toroidally over metre distances in the divertor plasma. Therefore, they would have been clearly detected by the IR cameras of the ASDEX Upgrade first wall protection system $[14,30]$.

For the sloped sample the volume of recessed melt zone versus melt residue elevated above the original surface level was determined to $4.1 \mathrm{~mm}^{3}$ for the valleys and $4.0 \mathrm{~mm}^{3}$ for the hills respectively. This supports quantitatively the impression gained by initial visual inspection that melt motion along the sloped surface was considerably reduced with no detectable net loss of material from the melt zone. This does not exclude ejection of minor droplets, which has been previously observed in ASDEX Upgrade, at least in case of sustained melting of leading edges [15]. The diameter of the ejected droplets in these earlier experiments was estimated to be smaller than $100 \mu \mathrm{m}$, which is not detectable in the 3D surface scans.

In summary, both the quantitative mapping of the post-melting surface morphology and the time point where material from the leading edge melt zone reached the gap between sample and surrounding tile provide a well-defined data set for validation of the MEMOS melt model, which is currently ongoing.

\section{Structural material changes}

The structural changes of both the near-surface bulk tungsten and the melt residue were examined by scanning electron microscopy (SEM) using a FEI HELIOS NANOLAB 600 microscope with an integrated focused ion beam (FIB) cutting unit.
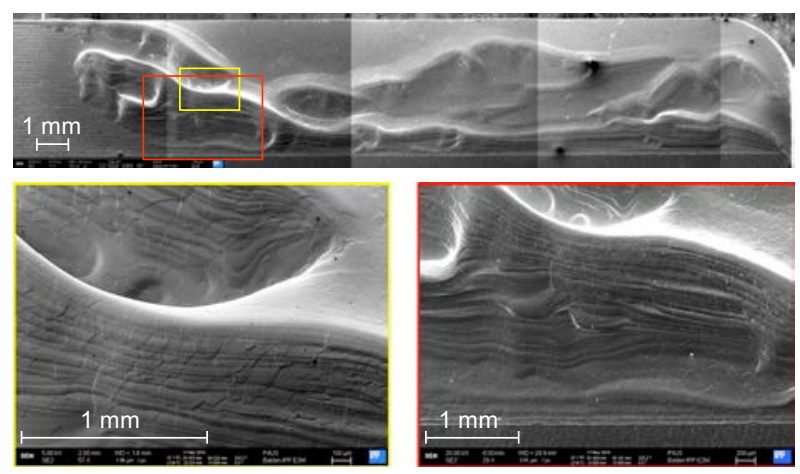

Fig. 10 SEM raster images of the sloped sample melt zone with magnified views of re-solidified melt debris.

Fig. 10 shows the surface morphology of the sloped sample melt zone with magnified views of re-solidified melt debris. The elevated melt residue has the appearance of a stratified structure, which suggests that it was formed in a step-wise pile-up process. However, the possibility that these features may instead be surface capillary waves frozen upon re-solidification cannot be excluded. Re-solidification also led to the growth of large crystal grains with a lateral size of up to $\approx 0.1 \mathrm{~mm}$. This suggests a much lower mechanical stability and embrittlement of the melt residue compared to the original bulk tungsten. One consequence of this might be to promote spallation of melt residue by mechanical stress induced in subsequent plasma exposure as observed in previous divertor studies of sustained tungsten melting [15].

The grain structure of the melt residue was further analyzed by FIB cuts on the leading edge sample across the interface between the original bulk-W surface and the melt residue depicted in Fig. 11. The latter exhibits large grains, similar in size to those seen on the sloped sample. The FIB cross-sections reveal that the grains also extend to the same size scale into the depth of the re-solidified melt trace.

In comparison, the depth of the transient melt layer computed with MEMOS for the JET leading edge experiment was $\approx 10 \mu \mathrm{m}[3,5]$. Assuming similar melt layer depth in the ASDEX Upgrade melt exposures, this underlines that the layered appearance of the melt residue along the leading edge samples should not necessarily be directly interpreted as sign of a step-wise melt transport process. The data suggest that the piled up debris was formed instead by zonal growth in the resolidifying melt layer seeded by the crystal planes at the liquid-solid interface. 

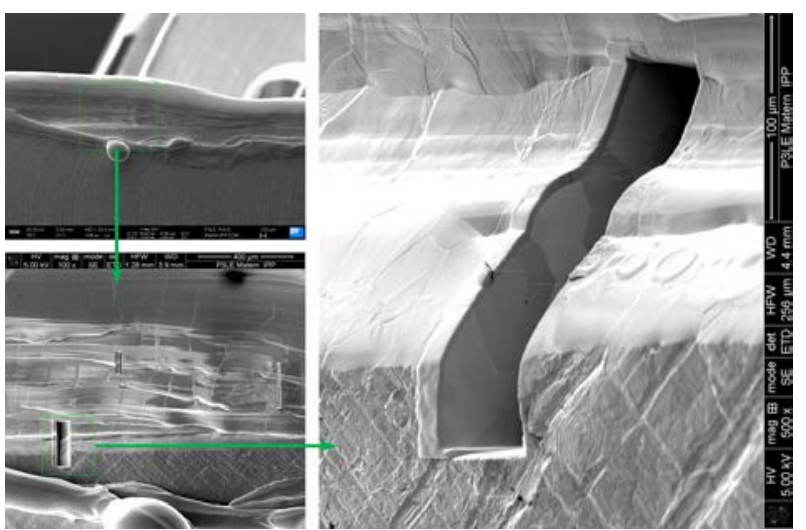

Fig. 11 SEM raster images of the re-solidfied melt debris at the lower part of the leading edge sample. The magnified images show the location and detailed view of a FIB cross-section at the interface between melt residue and original bulk tungsten surface.

Following SEM and FIB analysis, the leading edge sample was finally cut for metallographic analysis of the grain structure at cross-section surfaces. After cutting, the cross-sections were first polished and then etched to enhance the visibility of the grain structure. Fig. 12 shows the cross-sections with the position of the cuts. Shallow melting can already been seen on the crosssection near the upper poloidal edge of the sample (Fig. 12a) with the largest distance to the OSP during exposure. The two cross-sections below from the actual melt zone (Fig. 12b and c), show an approximately linear increase of the melt erosion depth with increasing height above the surrounding tile level. Fig. 12b shows that melting began actually at $\approx 1.2 \mathrm{~mm}$ below the top surface of the sample. Since the height of the leading edge is $1.1 \mathrm{~mm}$, this indicates that the plasma flux penetrates into the $\approx 1 \mathrm{~mm}$ wide gap between the main tile and the sample. Such gap edge loading is expected as a consequence of magnetic field line inclination coupled with finite ion Larmor radius and possibly the effects of sheath potential structure across the gap [31].

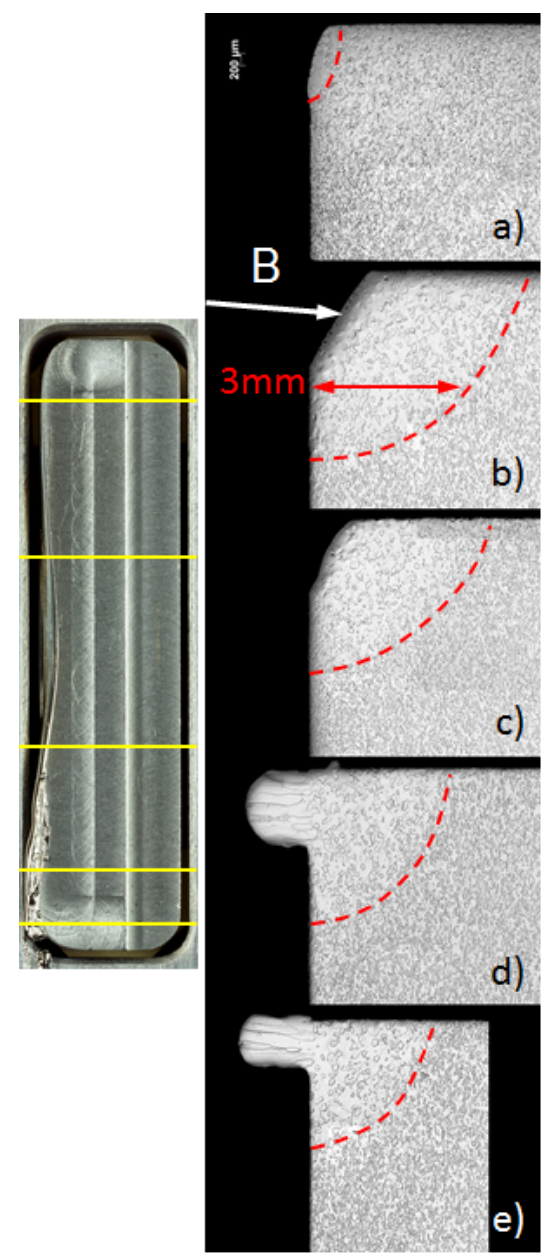

Fig. 12 Cross-sections through the leading edge sample along the toroidal coordinate with the plasma exposed edge at the left. The position of the cross-sections is marked by the yellow lines in the photo of the sample top surface. The dashed red lines indicate the approximate extent of recrystallization into the bulk material below the melt zone. The arrow shows the direction of magnetic field and of incident plasma flux.

The two cross-sections in Fig. 12d,e were made in the part of the sample which resided in the private flux region (PFR) during the melt exposures. The melt residue cross-section confirms the results of the FIB analysis, clearly showing the columnar structure of tungsten crystallites, interpreted as the result of zonal growth at the melt-solid interface, i.e. re-solidifying melt taking over the crystal structure and orientation of the substrate crystal grains. The high temperature of the sample also leads to significant re-crystallization of the original tungsten structure to depths of up to $\approx 3 \mathrm{~mm}$ in the melt zone with depth decreasing towards locations, which were exposed to lower overall power flux. This highlights that damage by repeated power transients may already occur below the melt damage threshold because the re-crystallization will lead to corresponding deterioration of the mechanical strength and ultimately to increased risk of crack formation. The latter might 
also increase the risk of further melting in following ELM transients.

\section{Power flux onto samples}

Analysis of the samples' thermal response and modelling of the transient melt processes using MEMOS rely on accurate input for both the spatial distribution and the temporal evolution of the power load onto the sample, including within each ELM. To reduce the complexity of the MEMOS simulations it is preferable to provide an analytical description of a representative ELM footprint instead of using the raw experimental power flux data from an actual sample exposure. Analytical expressions for the power flux distribution both in the inter-ELM phase and during the ELM transients derived in this section were used for first MEMOS simulations of the transient melting at the leading edge sample, which are discussed in another publication [29].

To determine the time resolved local power flux distribution to the melt samples from the surface temperature, an infra-red (IR) CCD camera was installed viewing the divertor manipulator probe tiles via an image guide connected to an objective lens located behind the inner heat shield tiles. The transmission properties of the glass fiber bundle limit the observable spectral range to near-IR wavelengths from $0.9-2 \mu \mathrm{m}$. Visual range light emission and IR emission below 1.6 $\mu \mathrm{m}$ are blocked by an interference filter. Unfortunately the local IR system failed in the first discharge of the experiment reported here and was replaced for the remainder of the experiment day by a visual range CCD camera with $120 \mathrm{~Hz}$ frame rate to capture at least qualitatively signs of melting on the samples and potential ejection of droplets. As a consequence, the power flux onto the melt samples could only be derived by an indirect measurement using the standard outer divertor thermography diagnostic, viewing the outer target in the torus sector opposite to the divertor manipulator. Toroidal symmetry must thus be assumed when applying the heat flux density derived from this measurement to the sample.

\subsection{Power flux to the outer target plate}

The ASDEX Upgrade standard thermography diagnostic is based on a customized calibrated IR camera with a programmable real time data acquisition system, which provides adaptive control of both exposure time and frame rate as function of maximum recorded intensity $[32,33]$. During the melt experiment, the camera was operated with a toroidally limited region of interest and a correspondingly increased sampling rate of $2 \mathrm{kHz}$ to resolve ELM power transients in the ms range.

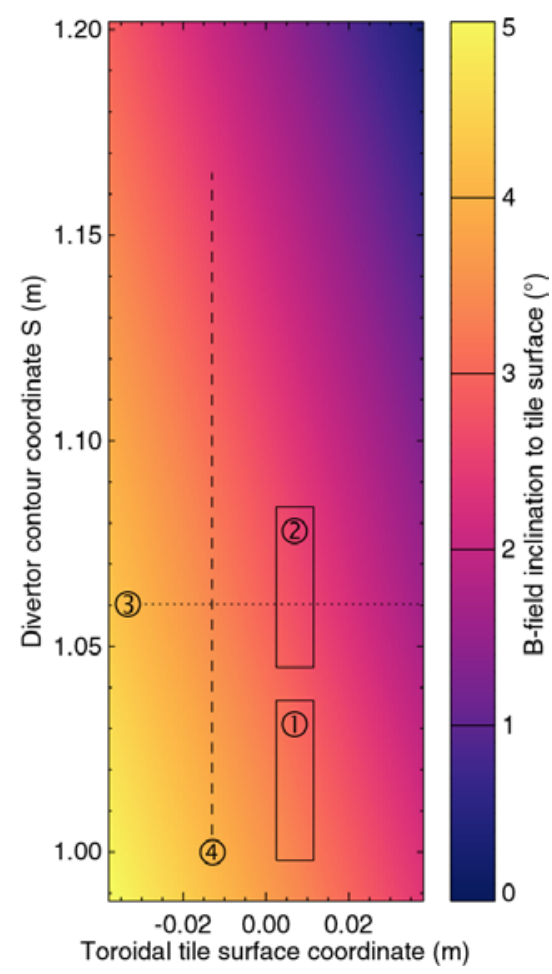

Fig. 13 Map of the field line angle of incidence to the flat divertor tile surface with contours of leading edge sample (1), sloped sample (2), outer strike line during slope exposure phase in discharge 33511 at $4.045 \mathrm{~s}$ (3) and poloidal contour used for thermography power flux density analysis (4).

The surface temperature is derived from the calibrated IR intensity with a spatial resolution of $0.5 \mathrm{~mm}$. With this input the power flux to the surface $P \operatorname{IR}(s, t)$ is computed along a vertical line $13 \mathrm{~mm}$ left of the tile center (Fig. 13) by a 2D (depth and poloidal coordinate) implementation of the THEODOR code [34]. To derive the local power flux at the sample location, the ASDEX Upgrade divertor tile geometry must also be carefully accounted for. Since the tile surfaces are flat, the magnetic field line incidence angle varies both poloidally and toroidally (see Fig. 13). The parallel heat flux, $P_{\|}(\mathrm{s}, \mathrm{t})$, is thus first derived from $P_{\mathrm{IR}}$ according to $P_{\|}(s, t)=P_{\mathrm{IR}}(s, t) / \sin \alpha_{\mathrm{IR}}(s, t)$ where $\alpha_{\mathrm{IR}}(s, t)$ denotes the local field line angle of incidence at the location of the IR evaluation. From $P_{\|}$the heat flux to the samples is then computed using the reverse geometrical projection with the local field line to surface angle $\alpha_{S}(s, t)$ at the sample location. A further issue for the power flux analysis are artefacts in the IR camera images, which originate on the one hand from superimposed reflections of stray IR light at the tungsten surfaces and on the other are attributed to the bremsstrahlung continuum emitted in the divertor plasma volume during transient detachment caused by the recycling neutral atoms released by the ELM plasma burst [23]. Since the IR camera views the target plate at a shallow angle with an almost toroidal line of sight across the divertor plasma, 
the bremsstrahlung fraction can transiently dominate the IR signal.

Without correcting for these artefacts, THEODOR analysis will initially compute an excess power flux because the artefacts will be interpreted as an increased surface temperature. Once they subside and the IR intensity reverts to representing the true local surface temperature, THEODOR will temporarily deliver a negative power flux to maintain energy conservation. This effect needs to be taken into account for correct assessment of the individual transient ELM power loads.

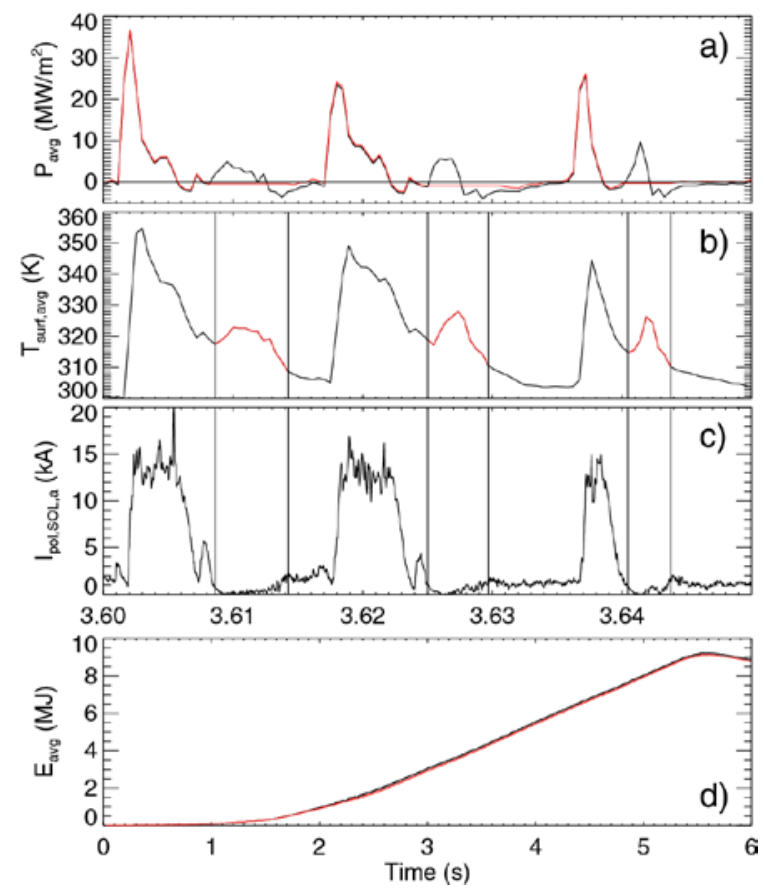

Fig. 14 a) Time traces of the average power load in discharge 33511 before (black curve) and after corrections (red curve) for three consecutive ELM cycles. b) Average tile temperature with identified IR volume radiation artefacts (red curve segments). c) Poloidal (thermoelectric) current from inner to outer divertor. d) Running integral of average surface power load in discharge 33511 before (black curve) and after corrections (red curve).

For detailed analysis of selected discharges the bremsstrahlung artefacts were identified by correlating total IR intensity with the poloidal thermocurrent between inner and outer divertor, measured by a shunt resistor [35]. Time intervals with detachment identified by vanishing poloidal current were manually cut from the IR data. Fig. 14 shows time traces of the average power load before and after corrections for pulse 33511 and also includes the average tile temperature with identified IR volume radiation artefacts. The poloidal current signal vanishes in the corresponding time intervals, also indicating temporary detachment due to the high recycling following ELM arrival.

While these corrections will improve analysis of the temporal evolution of an ELM cycle, they have a much smaller effect on the total energy delivered to the samples because, with integration over time, the excess power loads and subsequent negative power flux contributions approximately cancel out (Fig. 14d). The manual artefact correction was therefore omitted for those discharges where IR data were only required for comparison to the energy input derived from thermocouple measurements.

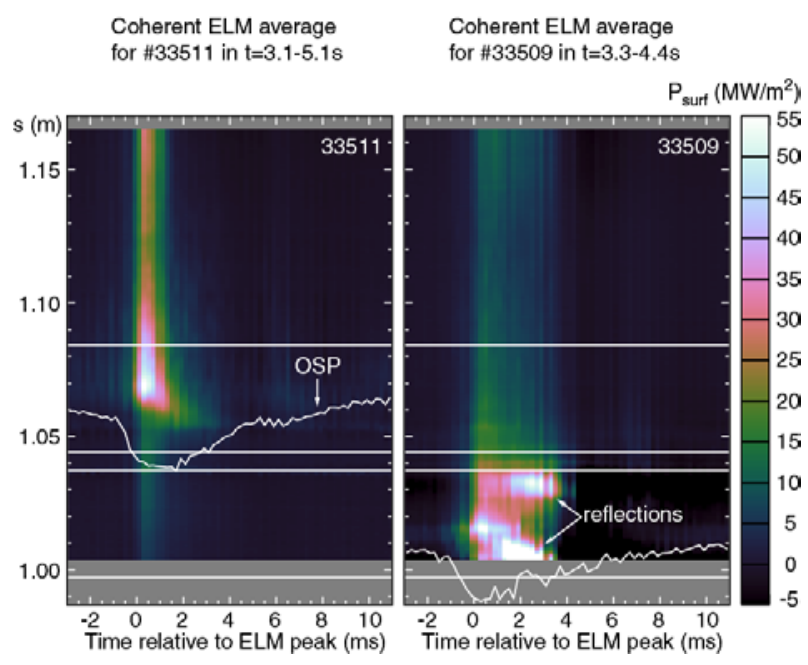

Fig. 15 ELM power flux footprint from IR-thermography. The data represent the coherent average of the ELMs within the sample exposure interval of discharges 33511 (sloped sample, $t=3.1-5.1 \mathrm{~s}$ ) and 33509 (leading edge sample, $t=3.3-4.4 \mathrm{~s}$ ). The y-axis reflects the poloidal divertor s-coordinate across the outer target tile starting at the lower tile edge at $s=0.987 \mathrm{~m}$. The horizontal lines denote the $s$-coordinate range of the two sample positions. Also shown is the coherent average of the OSP position from magnetic equilibrium reconstruction.

As input data for the derivation of the analytical description of the ELM footprint, all ELM transients during a given sample exposure interval were combined into a coherent average as shown in Fig. 15 for both sample geometries. The time window of $14 \mathrm{~ms}$ is given by the average ELM period. For the sloped sample exposure the position of the maximum power load agrees very well with the post-exposure melt pattern discussed in Section 3.1. Unfortunately, in contrast to the upper exposure location of the sloped melt sample, power flux analysis at the lower exposure position of the leading edge sample is hampered by strong reflections of the strike point IR emission from adjacent tiles (see Fig. 15). Moreover the lower sample position is not entirely covered by the viewing area of the thermography camera, which makes direct comparison with the rearside thermocouple measurements impossible. Therefore, as input for the MEMOS simulations in [29] only the ELM footprint measured at the upper sample location was used. For simulations of the leading edge sample the power flux distribution was merely shifted to the lower sample by the difference of the two OSP positions during exposure flat top. 
For the analytical description of the ELM footprint the power load is written as product $P(s, t)=E(s) \times f(t)$ of a spatial profile function, $E(s)$, and a normalized time evolution function, $f(t)$, where $s$ is the poloidal coordinate along the target plate. The shift of the magnetic equilibrium OSP position during the ELM peak (see Fig. 15), which leads to a corresponding small shift of the power flux maximum, is neglected in this approximation. The spatial profile is described by expression (2) in [36], which consists of a convolution of an exponential profile in the SOL region with a Gaussian to describe cross-field diffusion across the separatrix into the PFR [37]:

$$
\begin{gathered}
E(s)=\frac{E_{0}}{2} \\
\times \exp \left(\left(\frac{S}{2 \lambda}\right)^{2}-\frac{s-s_{O S P}}{\lambda}\right) \\
\times \operatorname{erfc}\left(\frac{S}{2 \lambda}-\frac{s-s_{O S P}}{\lambda}\right),
\end{gathered}
$$

where $E_{0}$ denotes the energy scaling factor, sosp the strike point position, $\lambda$ the SOL heat flux profile decay length and $S$ the divertor spreading factor.

The time evolution is approximated by expression (5) in [38], which is derived from the assumption of force free convective transport of particles along the open field lines in the SOL. Apart from the trivial time difference between analytical function and measured time evolution, $\Delta t_{\mathrm{ELM}}$, this model requires only the upstream parallel Mach flow number, $M_{\|}$and the characteristic free-streaming time, $\tau_{F S P}^{o}$, as free parameters. Because the energy scaling factor is already included in $E(s)$, the time evolution function is normalized to unity. It should be noted that the function is only to be taken for $t \geq 0$. This gives:

$$
\begin{aligned}
f(t) & \\
= & \frac{4}{2 \exp \left(-M_{\|}^{2}\right) M_{\|}+\left(3+2 M_{\|}^{2}\right) \sqrt{\pi}\left(1+\operatorname{erf}\left(M_{\|}\right)\right)} \\
& \times\left(1+\left(\frac{\tau_{F S P}^{o}}{t+\Delta t_{E L M}}\right)^{2}\right) \times \frac{\tau_{F S P}^{o}}{\left(t+\Delta t_{E L M}\right)^{2}} \\
& \times \exp \left(-\left(\frac{\tau_{F S P}^{o}}{t+\Delta t_{E L M}}-M_{\|}\right)^{2}\right) .
\end{aligned}
$$

Note that the normalization factor in eqn. (2) differs from that in [38] where for normalization $M_{\|}=0$ was assumed.

For the coherently averaged ELM footprint of the sample exposure interval in discharge 33511 from $t=3.1-5.1 \mathrm{~s}$ the spatial profile function was fitted to the measured profile of the ELM energy delivered to the surface within the ELM period. The latter was computed by integrating the ELM footprint data shown in Fig. 15 over time. The resulting fit as demonstrated by Fig. 16 matches the data very well. Apart from the shape itself, it should also be noted that the OSP position from the IR data fit agrees very well with the corresponding position from the magnetic equilibrium reconstruction. In addition the total energy delivered to the entire outer target plate can be computed by integrating the profile function over the $s$-coordinate and multiplying by the toroidal extent of the plasma wetted tile surface. For discharge 33511 this yields $E_{\mathrm{ELM}}=25.9 \mathrm{~kJ}$ compared to the average plasma energy drop per ELM of $42.2 \mathrm{~kJ}$ for the same discharge time interval from the magnetic equilibrium reconstruction. This is consistent with the rough expectation that approximately one half of the total ELM energy loss should be deposited at the outer target plate $[24,25]$.

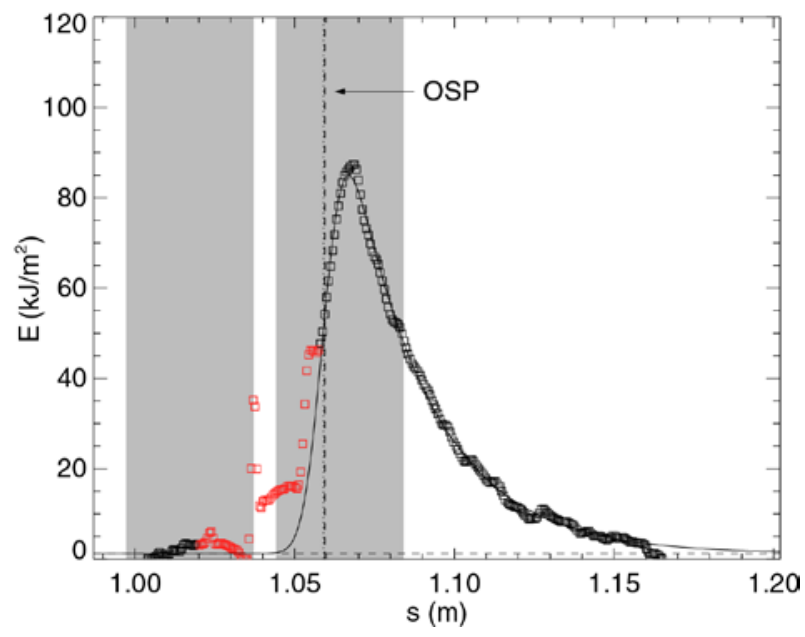

Fig. 16 Profile of the average total ELM target energy density derived from IR-thermography as function of the poloidal divertor s-coordinate. The data points represent the coherent average over the ELMs in discharge 33511 within the sample exposure time from $t=3.1-5.1 \mathrm{~s}$. The solid line represents the fit of the data to $E(s)$ in eqn. (1) with the parameters $E_{0}=123.6 \mathrm{~kJ}, s_{\mathrm{OSP}}=1.059 \mathrm{~m}, S=7.2 \mathrm{~mm}$ and $\lambda=24.8 \mathrm{~mm}$. Data distorted by reflections (red squares) were excluded from the fit. The shaded areas depict the poloidal extent of both sample positions and the vertical dotted line shows the OSP position from the magnetic equilibrium reconstruction.

It must be stressed, however, that the profile represents only the shape of the envelope over many subsequent ELMs, whereas the individual ELM profile will show a significant spatial variation due to its filamentary structure [24]. This needs to be taken into account for interpretation of simulation results because a given surface location of the modelled sample might, by using a coherently averaged ELM profile, be subject to transient power excursions with higher frequency than in reality, thus leading to a faster ratcheting up of local surface temperature by successive ELMs.

In a similar fashion as for derivation of the total ELM energy from the profile function, the experimental data for the time evolution of the total power load were 
obtained by integration over $s$ and multiplying by the toroidal extent of the plasma wetted tile surface. Fig. 17 shows the resulting data points, together with the fit of the time evolution function $f(t)$. As with the energy, the fit function describes the experimental data very well. Integration of the fit over the ELM period yields a second value of $E_{\mathrm{ELM}}=18.9 \mathrm{~kJ}$, providing a better match to the value expected from the average plasma energy loss per ELM. The discrepancy to the corresponding value obtained from the fit of the profile function is explained by the different constant background level in both fits.

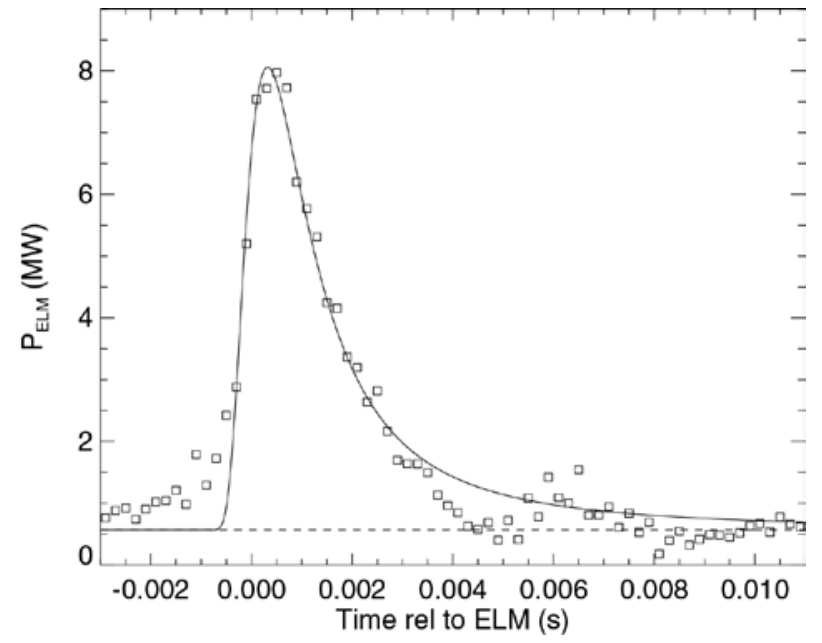

Fig. 17 Time evolution of the ELM power to the outer divertor target derived from IR-thermography. The data points represent the coherent average over the ELMs in discharge 33511 within the sample exposure time interval $t=3.1-5.1 \mathrm{~s}$. The solid line represents the fit of the data to $f(t)$ in eqn. (2) with the parameters $\tau_{F S P}^{o}=2.7 \mathrm{~ms}$ and $\Delta t_{\mathrm{ELM}}=1.4 \mathrm{~ms}$. In the fit the upstream Mach number was fixed at $M_{1}=0.5$.

In addition to the ELM power flux footprint, melt simulations also require the stationary profile of the inter-ELM surface power flux. This can be computed in similar fashion to the analysis of the ELM footprint by taking the conditional average of the power flux profile in a time window preceding the individual ELMs. Fig. 18a shows the resulting profile for the discharge of interest here. In the inter-ELM phases the power flux can also be extracted from an array of flush mounted Langmuir probes in the outer divertor. Assuming $T_{e}=T_{i}$, and following [39] (page 653, eqn 25.55):

$$
P=\left(\left(\gamma_{i}\left(1-R_{E}\right)+\gamma_{e}\right) T_{e}+E_{\mathrm{rec}}\left(1-R_{N}\right)\right) \times \Gamma_{i}
$$

where $\gamma_{i, e}$ denote the sheath transmission factors for ions and electrons, $R_{E}$ and $R_{N}$ the reflection coefficients for energy and ions at a tungsten surface, $E_{\text {rec }}$ the recombination energy of hydrogen ions to neutral atoms, and $\Gamma_{i}$ the ion flux derived from the ion saturation current.

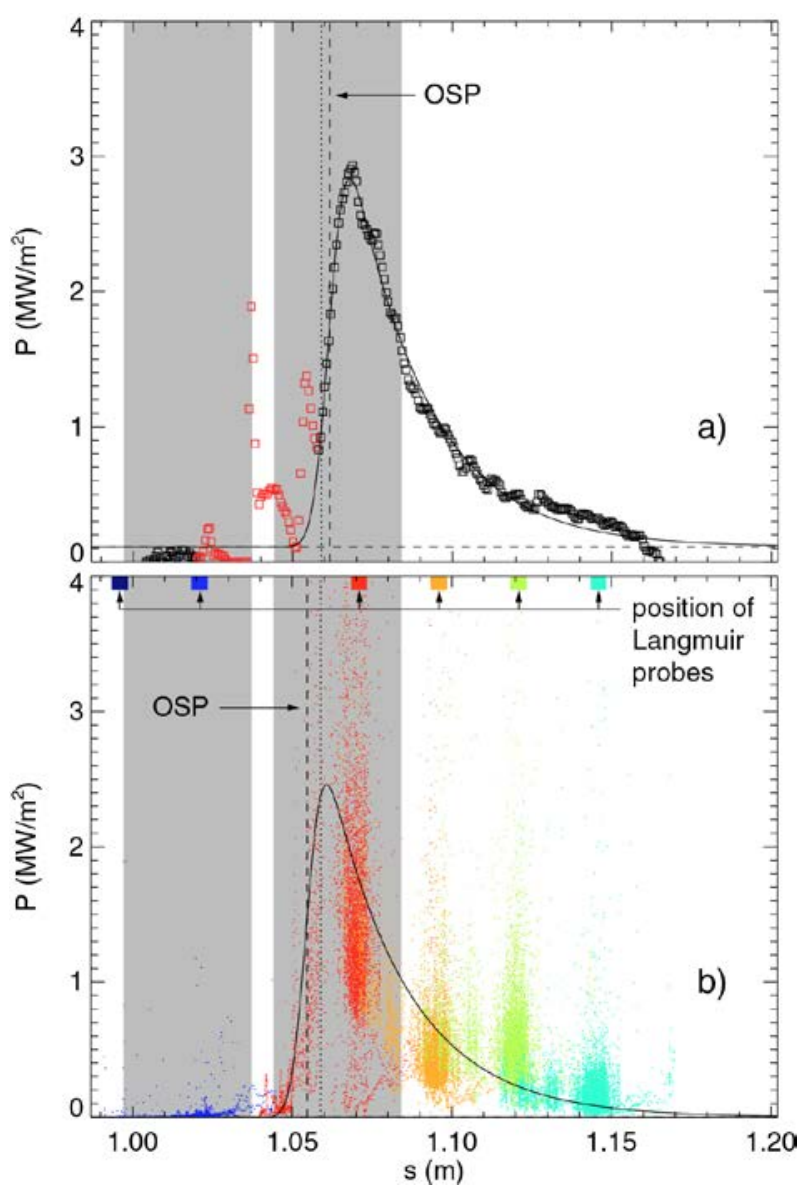

Fig. 18 Inter-ELM power flux density at target plates derived from IR-thermography (a) and Langmuir probes (b) as function of the poloidal divertor s-coordinate. The IR data points represent the coherent average over the pre-ELM time intervals 4-2 ms before ELM onset in discharge 33511 within the sample exposure time from $t=3.1-5.1 \mathrm{~s}$ (IR) and $t=2.7-4.4 \mathrm{~s}$ (Langmuir probes) respectively. The solid line represents the fit of the data to the profile function $P(s)$, analogue to the expression for $E(s)$ in eqn. (1) with the parameters $P_{0}=3.8 \mathrm{MW} / \mathrm{m}^{2}, s_{\mathrm{OSP}}=1.062 \mathrm{~m}, S=5.7 \mathrm{~mm}, \lambda=23.8 \mathrm{~mm}$ for the IR data and $P_{0}=3.4 \mathrm{MW} / \mathrm{m}^{2}, s_{\mathrm{OSP}}=1.055 \mathrm{~m}, S=5.4 \mathrm{~mm}$, $\lambda=24.4 \mathrm{~mm}$ for the Langmuir probe data. IR data distorted by reflections (red points in (a)) were excluded from the fit. The shaded areas depict the poloidal extent of both sample positions and the vertical dotted line shows the OSP position from the magnetic equilibrium reconstruction.

The resulting profile shown in (Fig. 18b) was derived from the data during the initial sweep of the OSP towards the sample position to improve spatial resolution. The results of the two entirely different diagnostics agree remarkably well (within $\sim 10 \%$ ) both in shape and magnitude.

\subsection{Comparison with thermocouple measurements}

Although, the power flux derived from thermography cannot fully substitute for the missing direct temperature measurement at the sample surface, it can be used to at least verify the resulting energy delivered to the samples by comparison with the temperature measurement at the rear surface of the samples. This is provided by 
thermocouples in contact with the sample rear surface, which acquire data both during the discharge and up to $200 \mathrm{~s}$ afterwards to monitor the cooling of the samples, due mainly to heat conduction through the bottom boronnitride pads used as electrical insulator. The heat transport through the sample smoothes out all transient surface power flux variations. Verification of the local power flux from IR-thermography data is therefore limited to comparison of the total energy input to the samples.

The energy input from IR-thermography data is obtained by integrating the total power flux to the samples. As described in section 5.1, the parallel power flux is first computed using the local B-field to surface inclination angle at the diagnostic location and then applied to the plasma wetted sample surface parts using geometric projection with the corresponding local inclination angle. The total power flux to the sample surface is then computed by integrating over the plasma wetted area. From the cross-section of the three sample types (Fig. 3) one obtains

$$
\begin{aligned}
P_{\text {flat }}(t)= & \int_{s_{\min }}^{s_{\max }} d s P_{I R}(s, t) \frac{\alpha_{0^{\circ}}(s, t)}{\alpha_{I R}(s, t)} \times 9 \mathrm{~mm} \\
P_{\text {slope }}(t)= & \int_{s_{\min }}^{s_{\max }} d s P_{I R}(s, t) \times \\
P_{\text {edge }}(t)= & \int_{s_{\min }}^{s_{\max }} d s P_{I R}(s, t) \times \\
& \left(\frac{\alpha_{0^{\circ}}(s, t)}{\alpha_{I R}(s, t)} \times 1.02 \mathrm{~mm}+\frac{\alpha_{15^{\circ}}(s, t)}{\alpha_{I R}(s, t)} \times 4.64 \mathrm{~mm}\right) \\
& \left(\frac{\alpha_{0^{\circ}}(s, t)}{\alpha_{I R}(s, t)} \times 5.5 \mathrm{~mm}+\frac{\alpha_{90^{\circ}}(s, t)}{\alpha_{I R}(s, t)} \times 1.1 \mathrm{~mm}\right)
\end{aligned}
$$

where $\alpha_{0^{\circ}}(s, t)$ is the local field to surface inclination angle at the flat part of the samples and $\alpha_{15^{\circ}}(s, t)$ and $\alpha_{90^{\circ}}(s, t)$ are the corresponding angles for the slope and leading parts respectively. The total energy input to a sample is finally obtained by integrating over the discharge time interval.

Fig. 19 shows the running integral of the input power to the samples for all discharges with melt exposures. For the samples at the lower exposure position (Fig. $19 b)$, i.e. the leading edge melt sample and its corresponding flat reference sample, it should be noted that the power input is slightly underestimated because of the in-complete coverage of the sample s-coordinate range by the IR diagnostic. The beginning of the melt exposure when the OSP arrives at the sample surface is indicated by the vertical black dashed line. The corresponding end times are indicated by similar lines in the color chosen for a given discharge. It is evident that even outside the actual exposure interval, when the samples are located in the PFR, the thermography data show a residual power load, which is larger for the upper sample position (Fig. 19a) because the OSP outside the exposure interval resides only slightly above the upper edge of the samples (see Fig. 7). This residual power load needs to be taken into account in the comparisons with the rear-side thermocouple temperature measurements. The final total input energies are listed in the legend with the values for the corresponding flat samples at the left tile in parentheses.

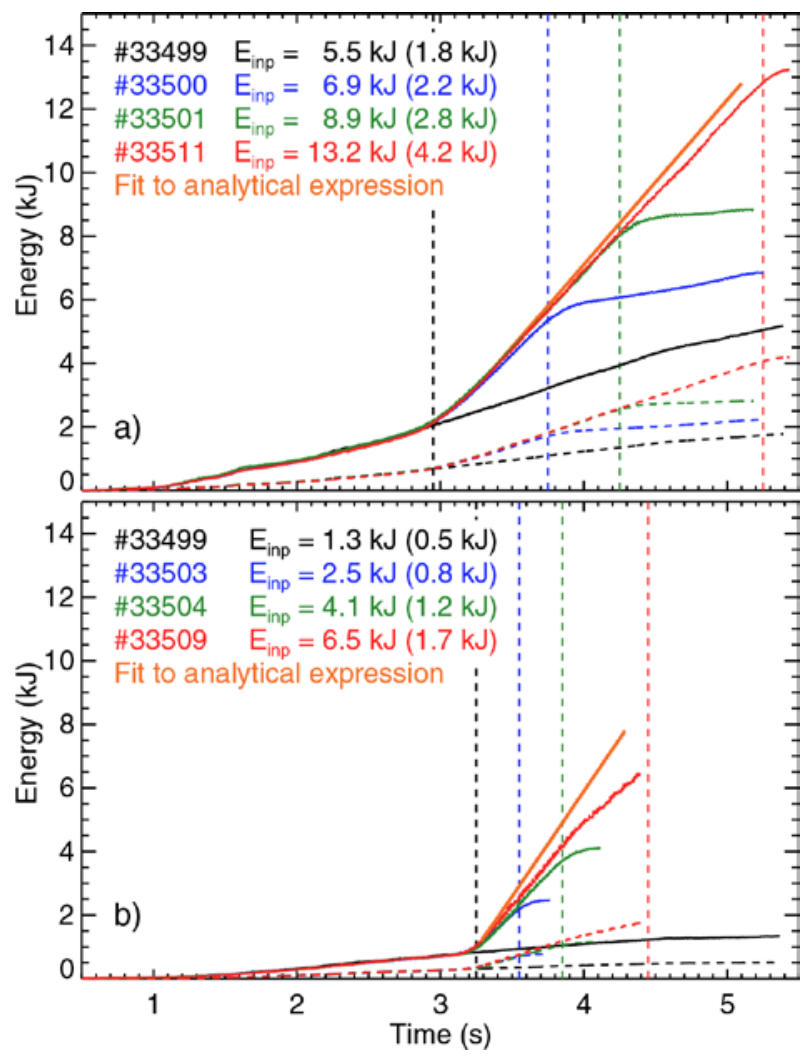

Fig. 19 Running integral of power input to samples from IR thermography for sloped sample (a) and leading edge sample (b). Solid lines refer to the actual melt samples, dashed lines refer to the corresponding flush mounted samples installed in the adjacent manipulator tile. Also shown is the power input computed from the analytical expressions for the time intervals of the sample exposure. For comparison the initial value was shifted to the corresponding experimental curve at the start time of the exposure indicated by the vertical black dashed line. The vertical dashed lines in the color chosen for a given discharge denote the respective time points when the OSP left the sample area.

The time evolution of the rear-side temperature is shown in Fig. 20 for the two melt samples. For comparison to the energy input from the thermography power flux, modelling of the heat transport from the sample surface to the support structure heat sink is required, and is planned for a forthcoming publication. However, in a preliminary, simplified analysis the exponential cooling curve can be fitted and extrapolated back in time to the end of the heating phase. The cooling time constant is a measure of the heat transfer coefficient between sample 
rear-side and support structure, which needs to be set accordingly in the detailed thermal models. The difference between the maximum post-discharge temperature and that at the beginning of the discharge determined in this way is then approximately proportional to the energy delivered to the samples during the discharge.

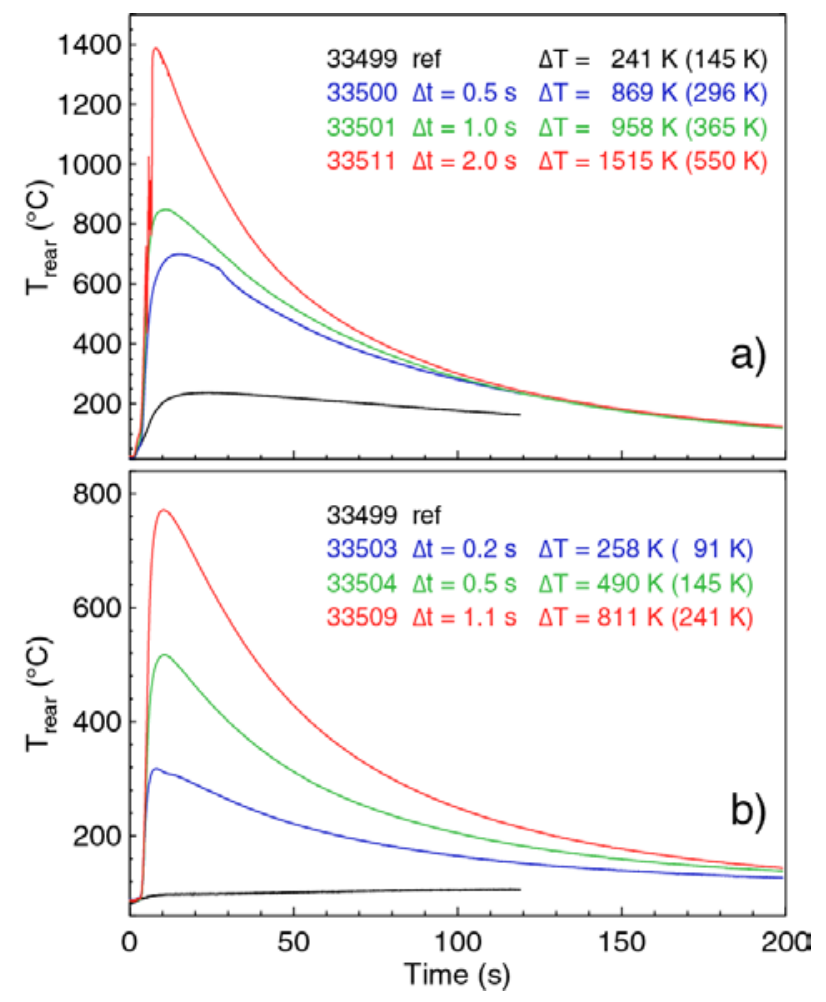

Fig. 20 Time evolution of the sample rear side temperature from thermocouple measurements for sloped sample (a) and leading edge sample (b). Deviations from exponential decay in some cases are caused by the thermal expansion of the tile frame during discharges, leading to an upward bend of the tile and corresponding loss of mechanical contact between TCs and sample rear side.

This is demonstrated by Fig. 21 a), which shows the ratio of temperature increase to sample energy input as a function of the strike point residence time on the different samples. Since the mass of the samples with protruding surface structures is slightly higher than their flush mounted counterparts, the respective values are normalized to the mass of the default flat samples. The approximately constant ratio of temperature increase versus integrated power flux confirms that the power flux from IR-thermography provides the correct energy input to the samples.

The ratio of energy input to the elevated samples to that of the flat samples can also be compared with the same ratio computed for the temperature increase of the samples. Since the computed energy input is derived from the power flux solely by geometric projection onto the sample surface and therefore only depends on the local magnetic field line to surface inclination angles, comparison with the sample temperature increase also allows the geometric (optical) projection approximation to be validated. As shown in Fig. 21b), the values for the samples agree within a $\approx 10 \%$ margin providing a clear demonstration of this validity.

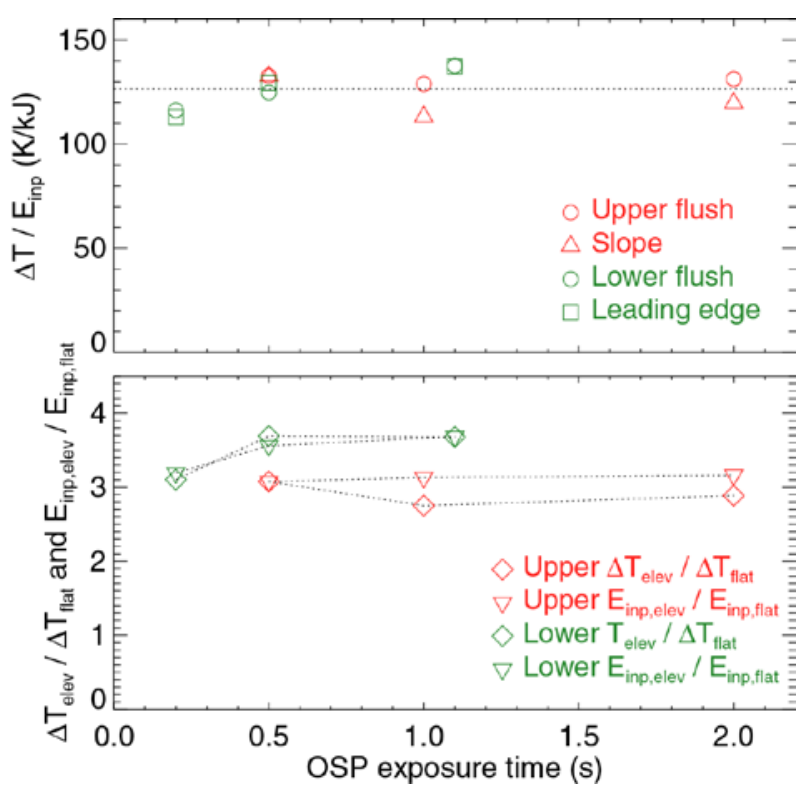

Fig. 21 a) Ratio of sample rear side temperature increase to total power input derived from surface power flux for the four samples. b) Ratio of total power input and of temperature increase of elevated samples to that of flush samples.

In summary the calorimetric analysis based on the thermocouple diagnostic confirms that the power flux to the samples can be reliably obtained by geometric projection of the power flux measured by the standard ASDEX Upgrade thermography system at a different toroidal position. Whilst this does not replace the missing local measurement of the sample surface temperature on an ELM-resolved timescale, melt modelling based on the available power flux is expected to provide a reliable estimate of the surface temperature, in turn allowing models used in the code for the thermionic emission current to be tested by comparison to the measured current discussed in the next section.

\section{Current flow through samples}

\subsection{Measurements}

The thermionic current which is expected to be emitted from a tungsten surface heated to above melting temperature cannot be quantified directly by measuring the corresponding replacement current from sample to vessel ground because of superimposed additional current fractions flowing between plasma and conducting surfaces. In the divertor these originate, apart from Pfirsch-Schlüter currents [40, 41], mainly from the thermoelectric effect due to the plasma temperature difference between inner and outer target plates [42, 43]. 
As described earlier, the resulting net poloidal current is measured routinely at ASDEX Upgrade, though that measurement is integrated over entire target tiles [35]. To determine the net replacement current assumed to be driven by thermionic emission from the melt samples, the local poloidal current fraction through the corresponding flat, flush mounted reference samples at the same poloidal position (Fig. 2) is subtracted from the total current through the melt sample. For this to be valid, the poloidal current distribution must be identical for identical sample geometry at both adjacent tiles. Fig. 22 demonstrates that this is indeed the case, showing that currents to identical flush mounted flat samples in both tiles match within the noise level.

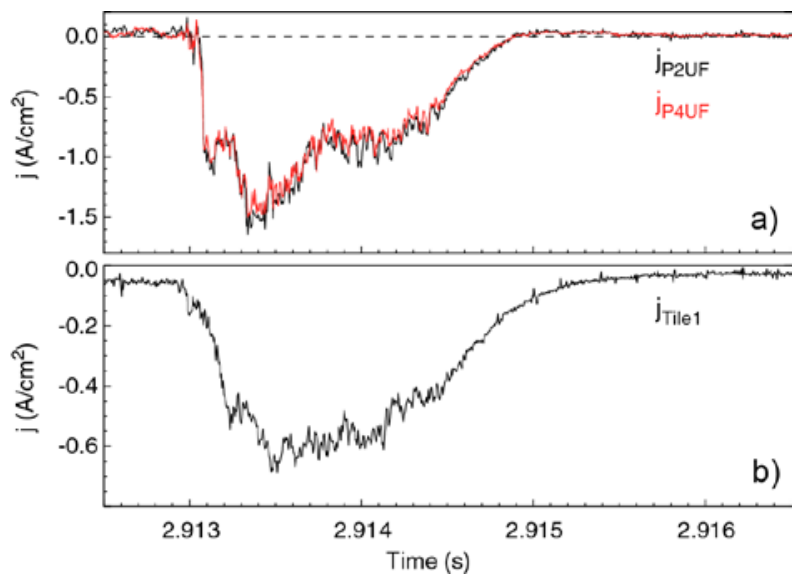

Fig. 22 Time traces of the local current density, given by the measured current divided by sample surface area, at the upper sample position during an ELM in H-mode discharge 33695 over a time interval where the sample position was above the OSP. The black curves represent signals from samples installed in the left, upstream, tile of the probe head while signals from the right, downstream, tile are plotted in red (see Fig. 2 for sample locations). For reference, the average current density through an entire tile is shown in (b).

The effect of the protruding surface structures on the melt samples must also be accounted for in assessing the sample current measurements since this modifies the effective current collecting area. The bulk tungsten samples have a length of $39 \mathrm{~mm}$ in the poloidal direction and a width of $9 \mathrm{~mm}$ in toroidal direction. Hence, the flat samples have a surface of $351 \mathrm{~mm}^{2}$. Assuming current flow along flux tubes to the sample surface, the melt samples will not collect a current at surface areas shadowed by the elevated features. For the leading edge sample this leaves an area of $5.5 \mathrm{~mm} \times$ $39 \mathrm{~mm}=214.5 \mathrm{~mm}^{2}$ facing the flat tile surface flux and a vertical area of $1.1 \mathrm{~mm} \times 39 \mathrm{~mm}=42.9 \mathrm{~mm}^{2}$ facing the parallel flux (Fig. 3). The current $I_{\text {edge }}$ through the leading edge sample can then be obtained by multiplying the current $I_{\text {flat }}$ through the flat sample with a geometric factor, which depends only on the local magnetic field line angle to the tile surface, $\alpha$ :
$I_{\text {edge }}=I_{\text {flat }} \frac{214.5+42.9 \cot \alpha}{351}$

The sloped melt sample has an elevated tilted surface with a plasma wetted toroidal extent of $4.3 \mathrm{~mm}$ at a slope angle of $15^{\circ}$ and a flat top part of $1.02 \mathrm{~mm}$ width (Fig. 3 ), resulting in an area of $1.02 \mathrm{~mm} \times 39 \mathrm{~mm}=39.8 \mathrm{~mm}^{2}$ exposed to the flat tile surface flux and an area of $4.3 \mathrm{~mm} \times 39 \mathrm{~mm}=167.7 \mathrm{~mm}^{2}$ receiving the flat tile surface flux magnified by a factor of $\sin \left(\alpha+15^{\circ}\right) / \sin \alpha$. This gives for the current $I_{\text {slope }}$ through the sloped sample:

$I_{\text {slope }}=I_{\text {flat }} \frac{39.8+167.7 \sin \left(\alpha+15^{\circ}\right) / \sin \alpha}{351}$

To check the validity of the effective current collection areas in equations (5) and (6), the sample currents must be measured in a plasma phase with sufficiently low heating power to avoid errors due to onset of thermionic emission. For the sloped sample this was possible during the melt experiment in the phase right after $\mathrm{x}$-point formation when the heating power was only $4 \mathrm{MW}$.

Fig. 23a) shows time traces of the measured currents in discharge 33500 in this initial low power phase. With a local magnetic field line to surface angle of $\approx 2.4^{\circ}$ in that time interval, equation (6) yields $I_{\text {slope }} \approx 3.5 \times I_{\text {flat }}$, which is about $50 \%$ above the measured factor of 2.15 . The discrepancy suggests that the current flow to samples with protruding regions does not depend solely on the field line to surface angle, but may, for example, be further modified by the influence of the surface shape on the local sheath potential. Quantitatively, these effects have been studied by means of particle-in-cell (PIC) modelling [44]. Corresponding studies are currently under way for the particular geometry and plasma conditions of the experiments discussed in this paper. In the absence of such simulation results, in what follows, the experimentally determined factor will be used to derive the net replacement current fraction attributed to thermionic emission, Inet, from the total current through the melt samples, Ielev, according to

$$
I_{\text {net }}=I_{\text {elev }}-2.15 \times I_{\text {flat }} .
$$

An example is shown in Fig. 23b) again for the sloped melt sample in the initial low power phase of discharge 33500. In between ELMs the power flux to the elevated sample surface is still too low to heat the slope surface to temperatures where thermionic emission becomes relevant. However, during the ELM at $1.187 \mathrm{~s}$ current emission in opposite direction to the poloidal current fraction appears at the elevated sample. The signal in Fig. 23b) is the difference between the current through the flush and sloped samples. It is clearly correlated with the ELM signature in the total tile thermocurrent (Fig. 
23c). This net replacement current is in the following assumed to be predominantly driven by thermionic electron emission although it has to be noted that there might be additional contributions due to other mechanisms such as secondary electron emission. Accurate quantification of the thermionic emission fraction will ultimately require both directly measured surface temperature and comparison to simulations, which also take into account a possible saturation of thermionic emission by space charge formation above the heated surface in presence of a magnetic field [45]. Indications for this effect were previously seen in melt studies at TEXTOR, where the current through an exposed tungsten test limiter probe was measured up to surface melt temperature [46]. Since the molten area can be estimated from the post-exposure melt pattern, a lower bound for the thermionic emission current density can be obtained from the net replacement current, providing a direct qualitative criterion to distinguish transient melting from sustained steady state melting. Quantitative analysis will, however, require integrated modelling of both the sample heating by the incident power flux and of the current emission from the surface as function of the local temperature.

The current flowing through the samples during the melt exposure phases is shown Fig. 24 for the two longest melt exposures of each sample type. The left (Fig. 24 a,b,c) and right (Fig. 24 d,e,f) columns show the data from exposure of the sloped and leading edge samples respectively, with the exposure intervals indicated by the position of the OSP with respect to the samples as shown in Fig. 24c and $\mathrm{f}$. In Fig. 24b (discharge 33511) and Fig. 24c (discharge 33509) the current flowing through the flat reference samples is plotted together with the corresponding integral poloidal current to the outer divertor (blue traces). For that the total poloidal current was scaled by the ratio of flat sample surface to the total divertor surface. It should be noted that this scaling is only an approximation because it does not take into account that due to shadowing of the upstream tile edges in the outer divertor the total plasma wetted divertor surface is somewhat smaller than the geometric value. During the exposure interval both current measurements are fully correlated and show current flow in the same direction. With the strike point above the samples, the current flows in opposite direction, because the temperature of the PFR plasma on the sample is now smaller than the average plasma temperature at the inner divertor target.

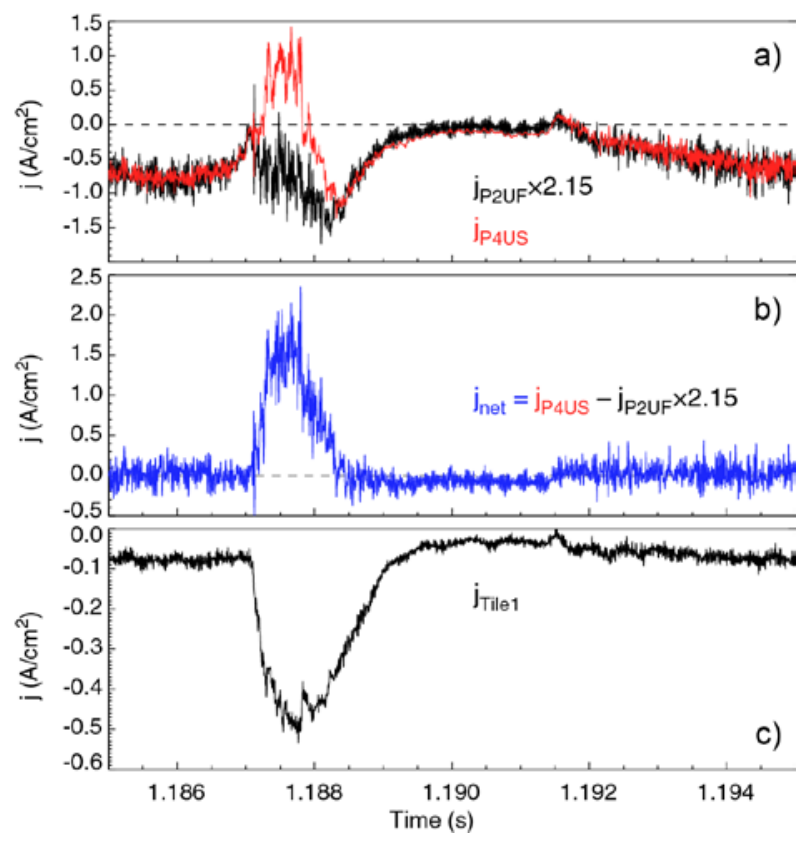

Fig. 23 Time traces of the local current density, given by the measured current divided by sample surface area, at the upper (a) sample position during an ELM in H-mode discharge 33500 measured at the flat flush mounted sample (black) and the sloped sample (red). Plot (b) shows the resulting net replacement current density attributed to thermionic emission. For reference, the average current density through an entire tile is shown in (c).

The net replacement current derived according to eq. (7) is plotted in Fig. 24a and d. The data are very reproducible between subsequent discharges. For both sample types, the onset of the current is coincident with OSP contact on the sample surface and the current flow subsides after the OSP leaves the samples again. At the sloped sample both the current in between ELMs and the current excursions during ELMs are initially approximately constant for $\approx 1 \mathrm{~s}$ before they begin to increase. This can be understood from the steep increase of thermionic emission with surface temperature. The start of the increase of the inter-ELM thermionic emission corresponds then to the time point at which the surface temperature distribution reaches a level sufficient to drive an integral thermionic current above the detection limit of $0.8 \mathrm{~mA}$. At this point ELM driven excursions will also begin to increase because each excursion in surface temperature will bring the surface further into the thermionic emission regime. Near the end of the exposure interval in discharge 33511 the current measurement at the melt sample begins to exceed the data acquisition range, indicated by the saturated ELM excursions in Fig. 24a,d. It should be noted that current excursions during ELMs are observed as soon as the OSP reaches the sample surface, even though the surface temperature is clearly below the level at which thermionic emission would be detectable. This indicates that a fraction of the measured current is driven by as yet 
unidentified mechanisms, which do not depend on surface temperature.

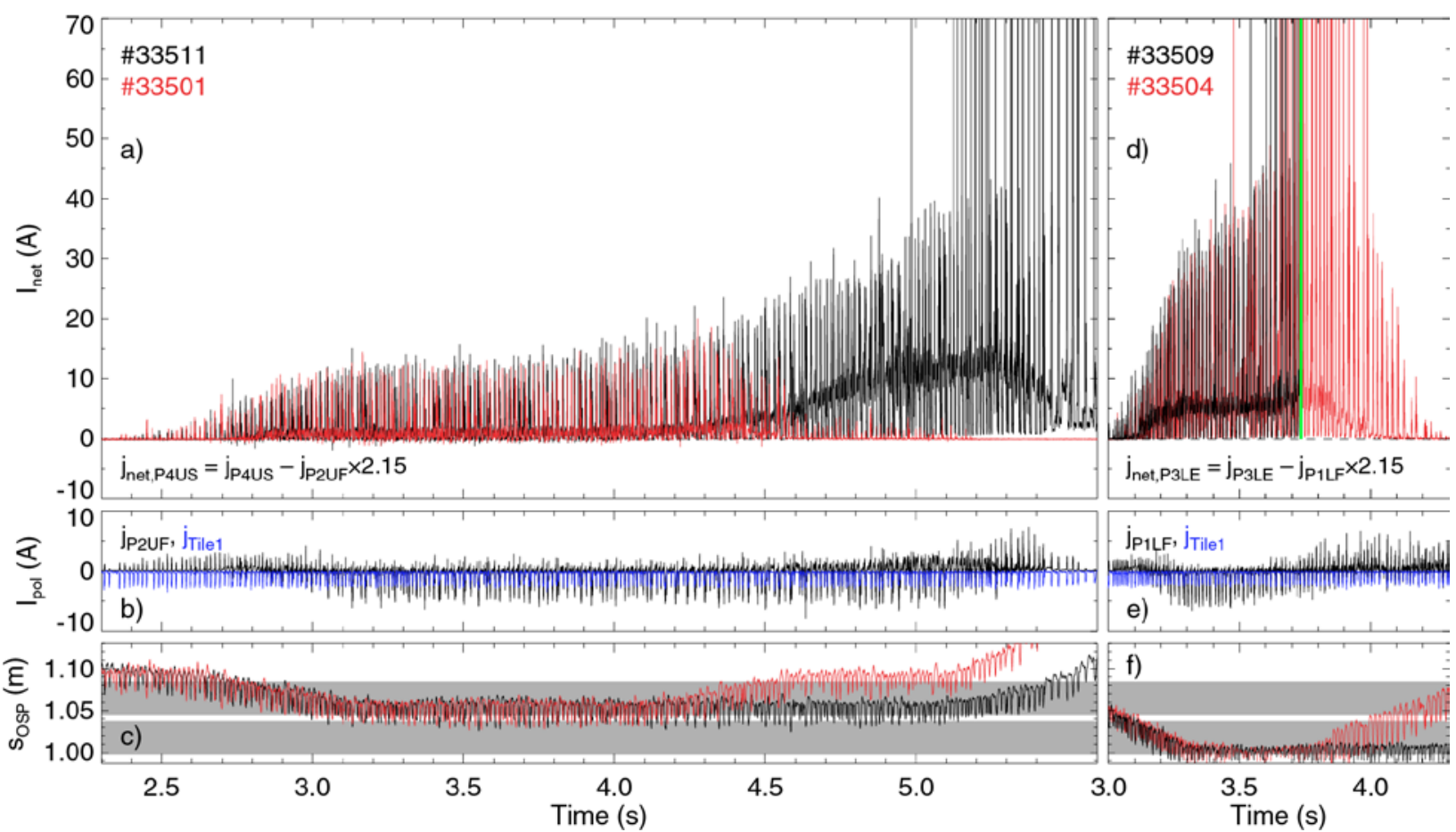

Fig. 24 a,d) Current density fraction through the melt samples to vessel potential attributed to thermionic emission. The left column shows the signals for two exposures of the sloped sample. The right column shows the signals for two exposures of the leading edge sample. b,e) Current density through flush reference samples and corresponding average poloidal current density to the outer divertor surface (blue trace). c,f) Position of outer strike point during melt sample exposures. The poloidal extent of the samples is denoted by the shaded areas.

Similar behavior is observed for the leading edge sample, although in this case the surface is heated much more rapidly to a temperature level where thermionic emission becomes significant. Correspondingly the ELM-related excursions of the net replacement current increase almost immediately, reducing only when the OSP moves away from the sample in discharge 33504. During the longer exposure in the last discharge of the series (33509), the current jumped to saturation level after the ELM at $t \approx 3.73 \mathrm{~s}$, indicated by the vertical green line in Fig. 24d, remaining there until the end of the exposure time interval. This is attributed to melt crossing the gap between sample and tile at that time point, shorting the sample to the tile and driving the entire tile current through the sample shunt resistor. The latter was ultimately destroyed by overload. Postexposure inspection of the probe head confirmed the established melt residue bridge between sample and surrounding frame tile (see Fig. 9d). Although the thermionic current could not be recorded to the end of the melt exposure, the accurate timing of the melt motion spilling over to the surrounding tile provides a welldefined additional boundary condition for the validation of future melt modelling.

The high time resolution of the current measurements also provides detailed information on the processes occurring within an individual ELM cycle. Fig. 25 shows the fine structure of the measured currents for the leading edge sample and its flat reference sample in discharge 33509. The fluctuations during each ELM are attributed to the passing of individual ELM filaments [25] and are routinely observed, for example, in tokamak divertor Langmuir probe measurements. This demonstrates that discrepancies must be expected between experiment and melt motion simulations based purely on a regular succession of a coherently averaged representative heat flux transient. 


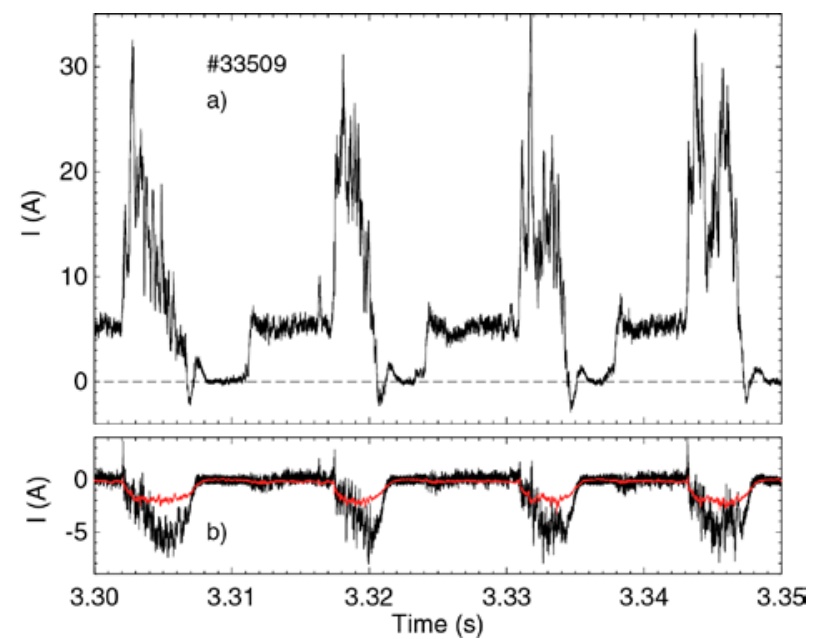

Fig. 25 ELM-resolved fine structure of current density through leading edge melt sample in discharge 33509 (a) and current density through flush reference sample and full divertor tile (red signal) respectively (b).

A second effect seen in the current is the observed drop after each ELM below the inter ELM level for a few milliseconds. A similar reduction is also observed in the total poloidal thermocurrent, which is attributed to the temporary detachment of the outer divertor due to the high recycling neutrals density after ELM impact [23, 47]. As discussed in section 5.1, this effect also leads to increased bremsstrahlung in the divertor volume, which is seen in the IR range by the thermography camera. The observed drop in the replacement current is not in contradiction to its origin from thermionic emission because even if the surface temperature does not decrease significantly in that short time interval, the resulting drop of electron temperature will lead to a corresponding drop of the sheath potential, which will in turn decrease thermionic emission by increased space charge formation.

\subsection{Comparison to Richardson-Dushman equation}

The measured replacement currents presented in the previous section immediately raise the question if, even without directly measured surface temperature, conclusions can be drawn regarding the melt processes. Foremost in that respect is the question of whether the observed melting occurred as a continuous ongoing process with the surface above tungsten melting temperature during the entire interval, or if, as intended in the experiment, melting took place as a repeated transient process driven by the ELM power excursions with the surface re-solidifying after each ELM. To resolve that, the measured thermionic current evolution may be compared with the current density of thermionic emission at a given surface temperature according to the Richardson-Dushman equation [48, 49],

$$
j_{t h}(T)=A_{R} T^{2} \operatorname{Exp}\left(-\frac{W}{k_{B} T}\right),
$$

with the Richardson constant, $A_{R}=6 \times 10^{5}$, Boltzmann constant, $k_{B}$, and tungsten work function, $W \approx 4.55 \mathrm{eV}$.

Since the measured currents through the samples represent integral values across the plasma exposed surface, to make the comparison it is further assumed that due to the strong increase of thermionic emission with temperature, the measured current will be entirely dominated by the hottest surface region. The sample areas affected by melting, determined post-exposure (section 3.2), can be used as an upper limit of the emission zone. The current densities derived by this simple model for inter-ELM and ELM phases are plotted in Fig. 26 in comparison to the temperature dependency according to equation (8). For the leading edge sample the inter-ELM value of the replacement current density is clearly below the value expected from the thermionic emission assumption at the tungsten melting temperature, while the peak value during ELMs is larger than expected at melt temperature. However, for the sloped sample, due to the considerably larger melt zone, the current density is in both cases below the value expected for tungsten melt temperature, in manifest contradiction with the observed melting. There are several possible explanations for this discrepancy. First, with increasing emission, space charge formation is expected [45], which reduces electron emission due to the corresponding reduction of the sheath potential. In addition, particularly for the sloped surface, melting was unlikely to have occurred homogeneously across the entire surface so that the local current density during the actual melt events will be higher than the value derived from the total post-exposure melt zone. The strongest difference between the leading edge and the sloped configurations is, however, the angle of the magnetic field intersecting with the surface. In case of the leading edge, the electrons emerging from the surface can move away freely along the field lines whilst in the sloped case (and thus even more so at the actual divertor target surface), a substantial fraction of the electrons is expected to be promptly redeposited at the surface due to the shallower field line incidence angle, which in turns reduces the net current flow (see Fig. 7 in [5]). This effect is of particular importance because it is expected to lead to a reduced melt motion at shallow field line to surface incidence and with lower resulting surface topological damage under transient loads. 


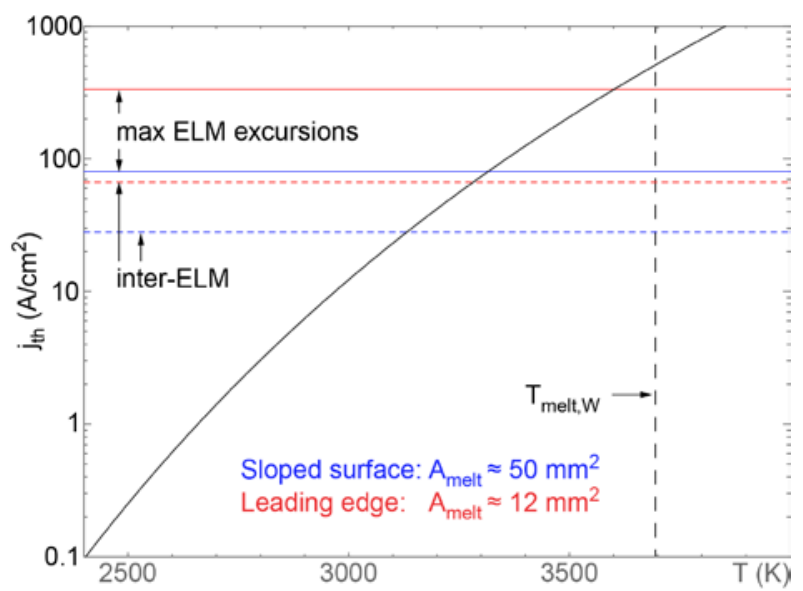

Fig. 26 Richardson current as function of temperature. The horizontal lines denote the estimates, based on post-exposure melt area, of the inter-ELM thermionic emission current (dashed lines) and during ELM transients near the end of the maximum exposure time intervals (solid lines) for both melt sample types.

In summary, at least in case of the leading edge sample, the measured net replacement current provides very strong evidence for repetitive transient melting, which is further supported by the close match of the resulting melt pattern to that observed under similar conditions in the previous JET experiment. In case of the sloped geometry, further assumptions are required to explain the lower thermionic emission current density. To obtain conclusive evidence for transient melting also in this case, the experiment will be repeated with additional local thermography of the surface to obtain a direct ELM-resolved measurement of the surface temperature.

\section{Conclusions}

The experiment presented in this paper was designed as companion study to the first JET transient ELM melting experiment [2-4] and was part of the ITPA Divertor and SOL Topical Group multi-machine coordinated task designed to assess leading edge power loading and melt dynamics [5].

One objective was to resolve the initial discrepancies encountered in the analysis of the surface power load to the leading edge of the special misaligned tungsten lamella used in the first JET experiment, which strongly violated the assumption of simple geometric projection of the parallel heat flux to obtain surface power flux density. Although the planned direct measurement of the sample surface temperature did not succeed in this first ASDEX Upgrade experiment reported here, thermocouples embedded in the exposed samples have allowed the derivation of the integral energy input for comparison with that extracted from power fluxes obtained with an IR camera system observing the divertor target at a distant location. The good match of both measurements for the different sample geometries confirms the validity of the geometric (optical) projection on ASDEX Upgrade, in agreement with the findings of the ITPA study. In fact, a rather significant effort mounted at JET has recently concluded that the initial discrepancies seen in the JET data can be resolved and that the optical projection ansatz also applies [19].

The main objective of the melt experiment reported here was the quantification of the thermionic emission current to provide further constraints for validation of the MEMOS melt motion model, which was invoked in the simulation of the JET experiment as origin of the observed melt motion drive. This has been confirmed in ASDEX Upgrade, in which the final melt pattern following exposure of a leading edge almost identical to that used in JET to a series of Type I ELMing H-mode discharges is remarkably similar to that seen at JET and in which the measured replacement current is clearly consistent both in magnitude and temporal behavior with transient melting correlated with the ELM power impulses. First attempts at modelling the ASDEX Upgrade data with MEMOS have also concluded that the measured replacement current is consistent with the observed melt motion [29]. Moreover, comparison of measured and predicted current provide strong evidence for the current being at least partly driven by thermionic emission, although there are clear indications for additional, as yet unidentified, mechanisms which show no clear correlation with surface temperature.

Exposure of a second sample, with a sloped geometry, revealed a radically different final melt pattern consisting of a corrugated surface clearly resulting from multiple re-solidification events and showing little or no net melt motion. The measured replacement current is also lower in comparison with the leading edge sample, though this could in part also result from an overestimated area of the transient melt zone at the sloped surface. Both the lower net current and the very different melt distribution support the assumption made in the JET MEMOS simulations that the escape of thermionic electrons is facilitated by geometries in which magnetic field lines impinge with near normal incidence [5], though MEMOS simulations of the sloped geometry have yet to be performed.

Post exposure, ex-situ analysis of the retrieved samples reveals recrystallization of tungsten at the exposed surface areas to a depth of up to several $\mathrm{mm}$. The melt layer transport to less exposed surface areas leads to a pile-up of re-solidified debris with zonal growth extending from the already enlarged grains at the surface. Independent of the current measurements the morphology in particular of the melt residue at the leading edge provides another strong indication for a ratcheting pile-up process during individual transient 
melt events. Both the recrystallization and the ongoing pile up of melt debris highlight the potential risk of repeated transient melting as well as of sustained excursions beyond thermo-mechanical material limits on the lifetime of affected plasma facing components.

\section{Acknowledgements}

The authors would like to thank the engineering and technical team, particularly Messrs. P. Leitenstern and Ch. Pfleger, for their support in preparing and running the experiment.

This work has been carried out within the framework of the EUROfusion Consortium and has received funding from the EURATOM research and training programme 2014-2018 under grant agreement No 633053. The views and opinions expressed herein do not necessarily reflect those of the European Commission or of the ITER organization.

\section{References}

[1] Herrmann, A., et al., A large divertor manipulator for ASDEX Upgrade. Fusion Engineering and Design 98-99 (2015) 1496-1499.

[2] Coenen, J.W., et al., ELM induced tungsten melting and its impact on tokamak operation. Journal of Nuclear Materials 463 (2015) 78-84.

[3] Coenen, J.W., et al., ELM-induced transient tungsten melting in the JET divertor. Nuclear Fusion 55 (2015) 023010 .

[4] Matthews, G.F., et al., Melt damage to the JET ITER-like Wall and divertor. Physica Scripta T167 (2016) 7.

[5] Pitts, R.A., et al., Physics conclusions in support of ITER $W$ divertor monoblock shaping. Nuclear Materials and Energy 12 (2017) 60-74.

[6] Bardin, S., et al., Evolution of transiently melt damaged tungsten under ITER-relevant divertor plasma heat loading. Journal of Nuclear Materials 463 (2015) 193197.

[7] Bazylev, B. and H. Wuerz, Melt layer erosion of metallic armour targets during off-normal events in tokamaks. Journal of Nuclear Materials 307 (2002) 69-73.

[8] Bazylev, B., et al., Experimental validation of $3 D$ simulations of tungsten melt erosion under ITER-like transient loads. Journal of Nuclear Materials 390-91 (2009) 810-813.

[9] Coenen, J.W., et al., Tungsten melt layer motion and splashing on castellated tungsten surfaces at the tokamak TEXTOR. Journal of Nuclear Materials 415 (2011) S78S82.

[10] Coenen, J.W., et al., Analysis of tungsten melt-layer motion and splashing under tokamak conditions at TEXTOR. Nuclear Fusion 51 (2011) 083008.

[11] Bazylev, B., et al., Numerical simulations of tungsten melt layer erosion caused by $J \times B$ force at TEXTOR. Physica Scripta T145 (2011) 014054.

[12] Bazylev, B. and J.W. Coenen, Modeling of Tungsten Melt Layer Erosion Caused by JxB Force at TEXTOR with the Code MEMOS. Problems of Atomic Science and Technology 83 (2013) 3-7.

[13] Coenen, J.W., et al., Evolution of surface melt damage, its influence on plasma performance and prospects of recovery. Journal of Nuclear Materials 438 (2013) S27$\mathrm{S} 33$.
[14] Krieger, K., et al., Controlled tungsten melting and droplet ejection studies in ASDEX Upgrade. Physica Scripta T145 (2011) 014067.

[15] Krieger, K., et al., Induced tungsten melting events in the divertor of ASDEX Upgrade and their influence on plasma performance. Journal of Nuclear Materials $\mathbf{4 1 5}$ (2011) S297-S300.

[16] Yang, Z., et al., 3D trajectories re-construction of droplets ejected in controlled tungsten melting studies in ASDEX Upgrade. Journal of Nuclear Materials $\mathbf{4 3 8}$ (2013) S846-S851.

[17] Arnoux, G., et al., Thermal analysis of an exposed tungsten edge in the JET divertor. Journal of Nuclear Materials 463 (2015) 415-419.

[18] Corre, Y., et al. Thermal analysis of transient tungsten melting experiments at JET. in 26th IAEA Fusion Energy Conference. 2016. Kyoto, Japan: IAEA-CN-234/EX/P65.

[19] Iglesias, D., et al., An improved model for the accurate calculation of parallel heat fluxes at the JET bulk tungsten outer divertor. Nuclear Fusion (2017) submitted.

[20] Coenen, J.W., et al., Transient induced tungsten melting at the Joint European Torus (JET). Physica Scripta T T170 (2017) 014013.

[21] Krieger, K., et al., Investigation of transient melting of tungsten by ELMs in ASDEX Upgrade. Physica Scripta T170 (2017) 014030.

[22] Schneider, P.A., et al., Pedestal and edge localized mode characteristics with different first wall materials and nitrogen seeding in ASDEX Upgrade. Plasma Physics and Controlled Fusion 57 (2015) 12.

[23] Laggner, F.M., et al., Divertor, scrape-off layer and pedestal particle dynamics in the ELM cycle on ASDEX Upgrade. Plasma Physics and Controlled Fusion (2017) accepted for publication, DOI: https://doi.org/10.1088/1361-6587/aa90bf.

[24] Eich, T., et al., ELM divertor peak energy fluence scaling to ITER with data from JET, MAST and ASDEX upgrade. Nuclear Materials and Energy 12 (2017) 84-90.

[25] Sieglin, B., et al., Assessment of Divertor Heat Load with and without External Magnetic Perturbation. Nuclear Fusion 57 (2017) 066045.

[26] Klimov, N., et al., Experimental study of PFCs erosion under ITER-like transient loads at plasma gun facility QSPA. Journal of Nuclear Materials 390-91 (2009) 721726.

[27] Garkusha, I.E., et al., Tungsten melt layer erosion due to $J x B$ force under conditions relevant to ITER ELMs. Journal of Nuclear Materials 363 (2007) 1021-1025.

[28] Makhlaj, V.A., et al., Damage of target edges in brushlike geometry in the course of ELM-like plasma pulses in QSPA Kh-50. Journal of Nuclear Materials 463 (2015) 210-214.

[29] Thoren, E., et al., Melt layer motion simulations with current constraints for an exposed tungsten edge in ASDEX Upgrade. Physica Scripta T T170 (2017) 014006.

[30] De Temmerman, G., et al., Melt-layer motion and droplet ejection under divertor-relevant plasma conditions. Nuclear Fusion 53 (2013) 8.

[31] Dejarnac, R., et al., Direct measurements of particle flux along gap sides in castellated plasma facing component in COMPASS. Fusion Engineering and Design 89 (2014) 2220-2224.

[32] Sieglin, B., et al., Real time capable infrared thermography for ASDEX Upgrade. Review of Scientific Instruments 86 (2015) 6. 
[33] Sieglin, B., et al., Real-Time Infrared Thermography at ASDEX Upgrade. Fusion Science and Technology 69 (2016) 580-585.

[34] Herrmann, A., et al., Energy flux to the ASDEX-Upgrade Divertor Plates Determined by Thermography and Calorimetry. Plasma Physics and Controlled Fusion 37 (1995) 17-29.

[35] Kallenbach, A., et al., Electric currents in the scrape-off layer in ASDEX Upgrade. Journal of Nuclear Materials 290 (2001) 639-643.

[36] Eich, T., et al., Inter-ELM Power Decay Length for JET and ASDEX Upgrade: Measurement and Comparison with Heuristic Drift-Based Model. Physical Review Letters 107 (2011) 4.

[37] Wagner, F., A STUDY OF THE PERPENDICULAR PARTICLE-TRANSPORT PROPERTIES IN THE SCRAPE-OFF LAYER OF ASDEX. Nuclear Fusion 25 (1985) 525-536.

[38] Eich, T., et al., On the asymmetries of ELM divertor power deposition in JET and ASDEX Upgrade. Journal of Nuclear Materials 390-91 (2009) 760-763.

[39] Stangeby, P.C., The Plasma Boundary of Nuclear Fusion Devices. Plasma Physics Series, ed. P. Stott and H. Wilhelmsson. 2000, Bristol and Philadelphia: IOP.

[40] Asakura, N., et al., Heat and particle transport of $S O L$ and divertor plasmas in the $W$ shaped divertor on JT60U. Nuclear Fusion 39 (1999) 1983-1994.

[41] Schaffer, M.J., et al., Pfirsch-Schluter currents in the jet divertor. Nuclear Fusion 37 (1997) 83-99.

[42] Chankin, A.V., Classical drifts in the tokamak SOL and divertor: Models and experiment. Journal of Nuclear Materials 241 (1997) 199-213.

[43] Staebler, G.M. and F.L. Hinton, CURRENTS IN THE SCRAPE-OFF LAYER OF DIVERTED TOKAMAKS. Nuclear Fusion 29 (1989) 1820-1824.

[44] Dejarnac, R., et al., Numerical evaluation of heat flux and surface temperature on a misaligned JET divertor $W$ lamella during ELMs. Nuclear Fusion 54 (2014) 12.

[45] Komm, M., et al., Simulations of thermionic suppression during tungsten transient melting experiments. Physica Scripta T (2017) in press.

[46] Sergienko, G., et al., Experience with bulk tungsten testlimiters under high heat loads: melting and melt layer propagation. Physica Scripta T128 (2007) 81-86.

[47] Wischmeier, M., et al., High recycling outer divertor regimes after type-I ELMs at high density in ASDEX Upgrade. Journal of Nuclear Materials 363 (2007) 448452.

[48] Richardson, O.W., The emission of electrons from tungsten at high temperatures - An experimental proof that the electric current in mitals is carried by electrons. Science 38 (1913) 57-61.

[49] Dushman, S., Electron emission from metals as a function of temperature. Physical Review 21 (1923) 0623-0636. 